\title{
Numerical and Experimental Insights into the Noise Generation of a Circulation Control Airfoil
}

\author{
L. Rossian* , A. Suryadi ${ }^{\dagger}$, K.-S. Rossignol ${ }^{\ddagger}$, R. Ewert ${ }^{\S}$, M. Herr ${ }^{\Uparrow}$ and J. W. Delfs ${ }^{\|}$ \\ German Aerospace Center (DLR), D-38108 Braunschweig, Germany \\ P. Kumar** \\ Institute for Fluid Mechanics, TU Braunschweig, Germany
}

With the advances in reduction of propulsion related noise from aircraft, airframe noise gets more and more into focus. During approach and landing, the high-lift system of the wings becomes one major acoustic source region contributing to the overall emitted noise. One promising approach to reduce this airframe noise is to change the complete high-lift system from a classic three element slat-wing-flap configuration to a slot-less system with active blowing and droop nose. Preceding experimental investigations have shown, that such a configuration may provide a noise reduction above $2 \mathrm{kHz}$ on the model scale. In the present paper both numerical and experimental investigations concerning the acoustics of a high-lift wing with droop nose and active blowing are presented. Thereby, an insight into the acoustic source mechanisms for different aerodynamic setups is provided that in the future will serve as a basis for the design of a low-noise high-lift configuration. It was found, that in principle three source mechanisms are to be considered. In the low to mid frequency domain, mostly turbulence-geometry interaction noise such as trailing edge noise, jet-nozzle interaction noise and curvature noise from the flow being bent around the flap are supposed to be the driving mechanisms. Moreover, the high frequency domain is found to be dominated by mixing noise from the high speed jet.

\section{Nomenclature}

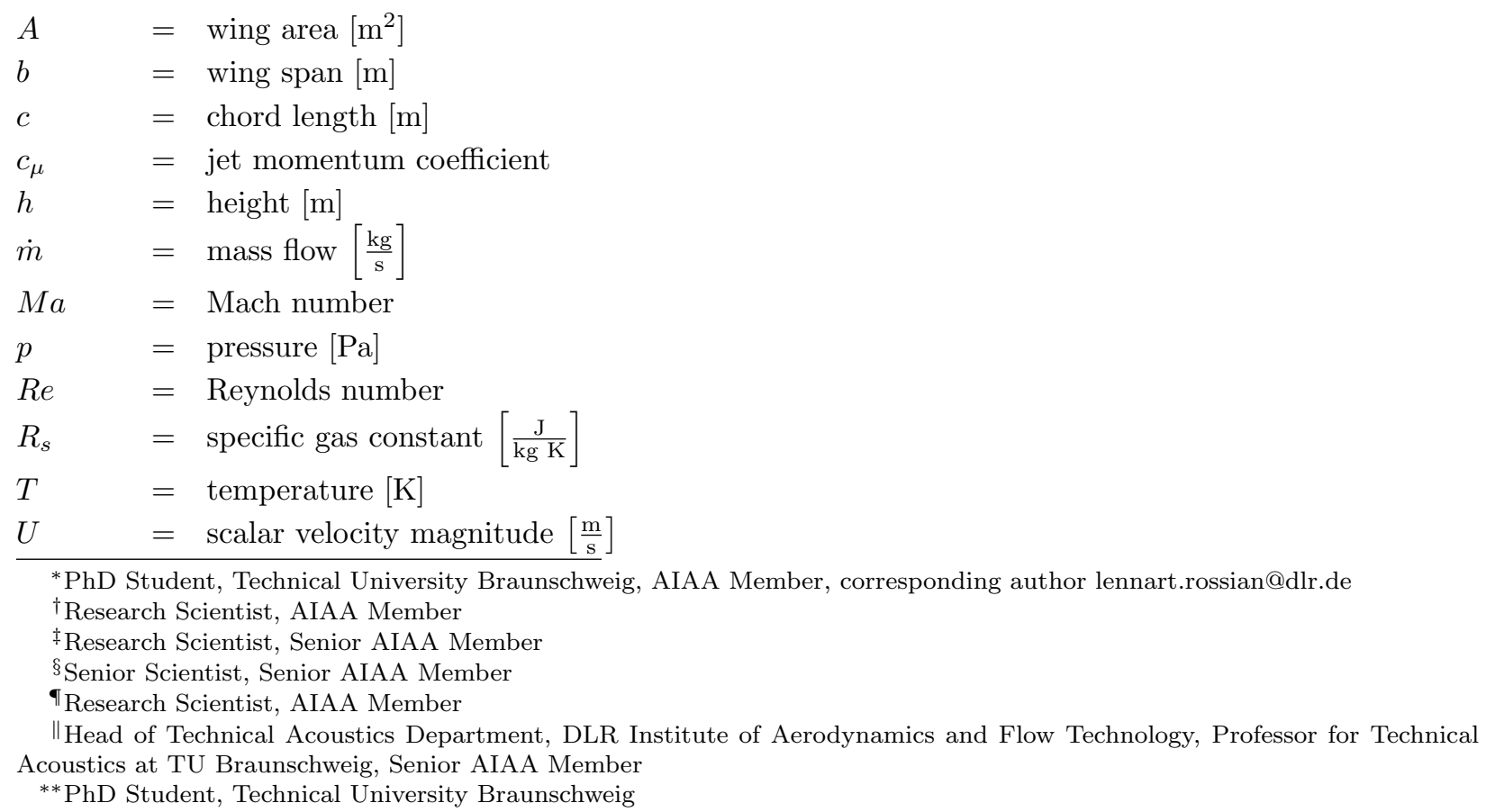




\begin{tabular}{|c|c|c|}
\hline$u$ & $=$ & velocity in $x$-direction $\left[\frac{\mathrm{m}}{\mathrm{s}}\right]$ \\
\hline$v$ & $=$ & velocity in $y$-direction $\left[\frac{\mathrm{m}}{\mathrm{s}}\right]$ \\
\hline$\alpha$ & $=$ & angle of attack $\left[^{\circ}\right]$ \\
\hline$\gamma$ & $=$ & adiabatic index \\
\hline$\rho$ & & density $\left[\frac{\mathrm{kg}}{\mathrm{m}^{3}}\right]$ \\
\hline$\sigma$ & $=$ & relaxation parameter \\
\hline$\omega$ & $=$ & vorticity $\left[\frac{1}{s}\right]$ \\
\hline \multicolumn{3}{|c|}{ Superscripts } \\
\hline 0 & $=$ & mean flow quantity \\
\hline ' & $=$ & fluctuating acoustic quantity \\
\hline \multicolumn{3}{|c|}{ Subscripts } \\
\hline$A W B$ & $=$ & Acoustic Windtunnel Braunschweig \\
\hline$\infty$ & $=$ & far field quantity \\
\hline jet & $=$ & blowing jet quantity \\
\hline$M U B$ & $=$ & Modell Unterschallwindkanal Braunschweig \\
\hline plenum & $=$ & plenum of the blowing jet inside the airfoil \\
\hline slit & $=$ & jet slit geometry \\
\hline
\end{tabular}

\section{Introduction}

The aim of the Collaborative Research Center CRC880 is to investigate concepts for high lift devices for future commercial aircraft. ${ }^{1}$ Therein, both aerodynamic and aeroacoustic analysis is carried out to meet performance and noise requirements. One promising approach to such low-noise high-lift devices are circulation control airfoils with high flap deflection angles as sketched in figure 1. These come without slotted slats and flaps and are able to provide very high lift coefficients. To avoid flow separation due to the strongly bend flow, a high speed tangential blowing is realized on the flap. This effect was first described by Coanda ${ }^{2}$ and has since been successfully simulated and tested in wind tunnels ${ }^{3}$ as well as flight demonstrators. ${ }^{4}$

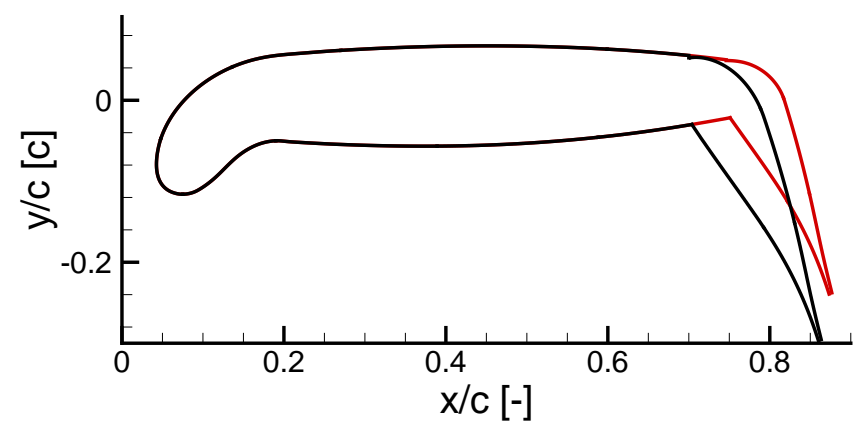

Figure 1: The two different airfoil geometries used for measurement and simulation (red) and simulation only (black) with reference clean chord length of $c=0.3 \mathrm{~m}$.

In 2010, Pott-Pollenske and Pfingsten ${ }^{5}$ showed experimentally that with such a circulation control airfoil significant noise reduction in the frequency range of 2 to $20 \mathrm{kHz}$ on model scale can be achieved compared to a classic 3 element high lift airfoil with slotted slat and flap. However, in the lower frequency domain a noise increase was found. It was conjectured but could not be concluded whether this increase might be related to wind tunnel installation effects or partially separated flow on the airfoil.

An analytical approach to break down the acoustic sources of a circulation control wing by Howe ${ }^{6}$ revealed four principle source mechanisms:

- interaction of jet turbulence with the jet slot 
- interaction of the exterior turbulent boundary layer with the jet slot

- curvature noise at the rounded edge behind the jet slot

- separation noise at the trailing edge

Note, that this analysis was carried out for an airfoil without flap but with a simple convex trailing edge. Therefore, transfered to the configuration in the present work, the separation noise will be replaced by classic trailing edge noise and the large separation bubble at the kink of the pressure side of the flap needs to be considered.

For the present work, both numerical as well as experimental investigations were performed on circulation control airfoils with a droop nose an a plain flap that are based on DLR's F16 airfoil, allowing for transonic cruise flight conditions. As sketched in figure 1, two different geometries were numerically addressed that differ in the extension of the flap (25\% chord length / $30 \%$ chord length). For the measurements, only the airfoil with the long flap was tested, whereas the short flap configuration will be addressed in future measurements. Additionally to the geometric difference, the simulations were run for various mean flow characteristics. These include changes in the angle of attack $\alpha$ and the blowing jet velocity $M a_{\text {jet }} /$ jet momentum coefficient $c_{\mu}$. Thereby a closer insight into the impact of partially detached flow on the airfoil's flap is possible. Within the experiments, a variety of parameters was tested, taking into account the free stream velocity, angle of attack and blowing jet velocity. By the combination of the simulation and experimental results, a detailed insight into the acoustic source mechanisms of an airfoil with active high-lift system can be delivered.

\section{Numerical Setup}

To simulate the noise radiated from an airfoil with blowing for circulation control a hybrid two-step CFD/CAA procedure with DLR's Finite Differences CAA Code PIANO ${ }^{7,8}$ is used. Therefore, in a first step an unsteady RANS CFD computation is run. From the result a time-averaged flow field is derived which provides the mean flow and the turbulence statistics. In the CAA step the Linearized Euler Equations (LEE) are solved atop of this mean flow. For this purpose, the acoustic sources on the right-hand side are provided by the fast Random Particle Mesh method (fRPM) by temporal reconstruction of the turbulent vortices based on the turbulence statistics provided by the CFD. ${ }^{9}$ In the following sections the basic input for the CAA computations is presented before going into detail about the considered acoustic source mechanisms.

\section{II.A. CAA Mesh and Mean Flow}

DLR's CAA code PIANO solves perturbation equations on a block structured mesh with a 4th order DRP scheme for discretization in space ${ }^{10}$ and a temporal 4th order Runge-Kutta scheme. The general topology of the $2 \mathrm{D}$ mesh can be seen in figure 2 along with different source regions that are linked to the source mechanisms presented in section II.B.2. The mesh is set to resolve frequencies up to $15 \mathrm{kHz}$ with 7 points per wavelength. Due to necessary refinements inside the source regions frequencies up to $30 \mathrm{kHz}$ are resolved on a circle with the radius of 1.5 chord lengths around the flap trailing edge. For the region of the blowing at the flap the mesh needs special refinement to resolve the jet that exits through a nozzle of $0.2 \mathrm{~mm}$ and reaches local Mach numbers on the order of 1 . With this refinement, the mesh consists of 2.7 million grid points in total.

For the acoustic simulations, two different setups were evaluated. These differ in three single aspects

- extension of the flap (setup 1: $30 \% \cdot c, \operatorname{setup~2:~} 25 \% \cdot c$ )

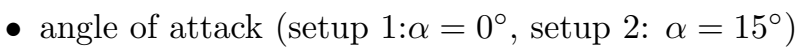

- velocity of the blowing jet (setup 1: $M a_{\text {jet }}=0.9$ resp. $c_{\mu}=0.062$, setup 2: $M a_{\text {jet }}=1.1$ resp. $\left.c_{\mu}=0.058\right)$

while the free stream Mach number of $M a_{\infty}=0.15$, and the Reynolds number of $R e=1 \cdot 10^{6}$ are kept constant. Figure 3 shows the two states of the mean flow around the airfoil by the local Mach number and selected streamlines calculated with the Reynolds Stress Equation Model (RSM). Here, two main aspects can be observed. First, for the case with high angle of attack the flow separates partially on the flap. Note, 


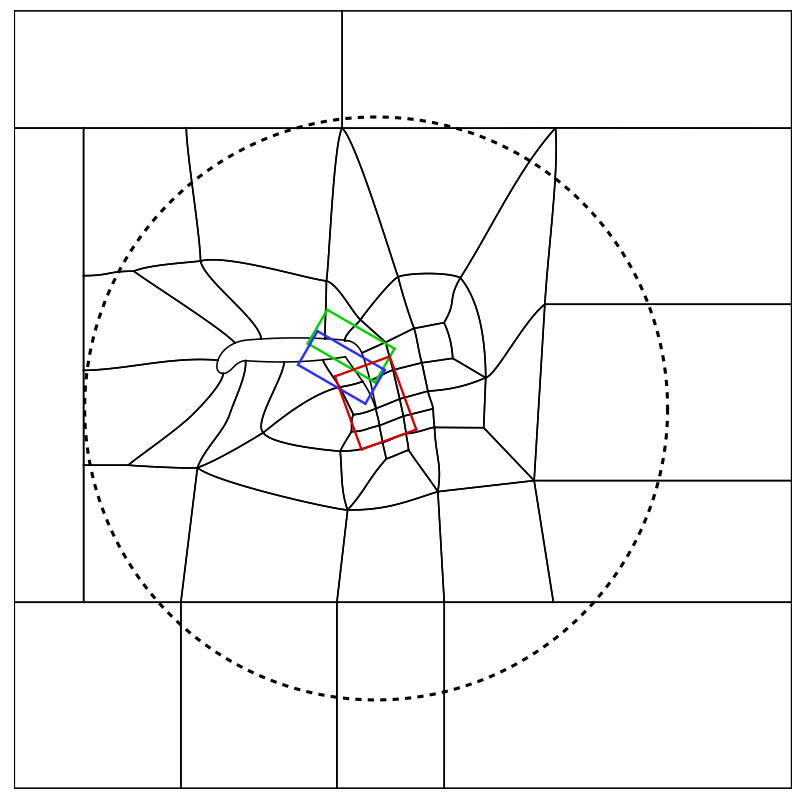

(a) Block structure of the CAA mesh with fRPM source regions marked by the colored blocks (red: trailing edge noise, green: curvature noise, blue: separation noise) and the microphone positions (dashed circle)

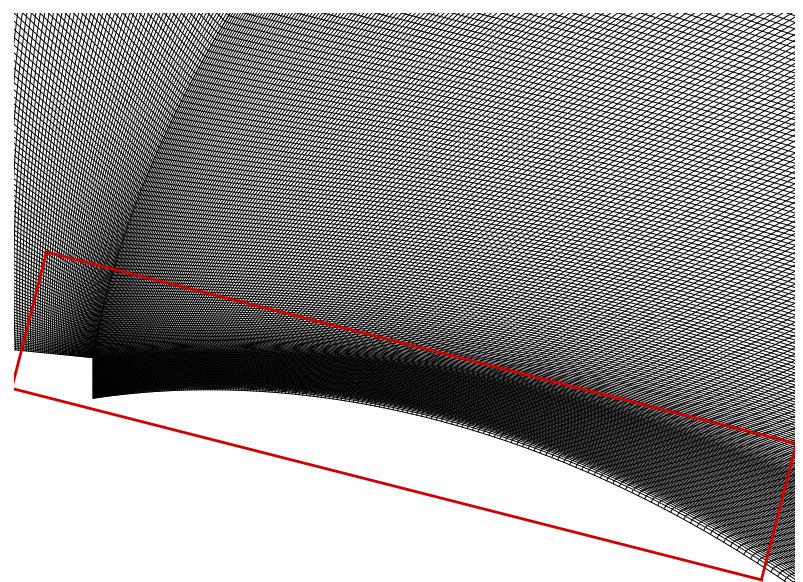

(b) Detail of the mesh refinement in the jet area with fRPM source region for the jet mixing noise (red box)

Figure 2: Structure of mesh used for the acoustic simulations.

that below the separated flow regime the wall-bound jet is still attached down to the airfoil's trailing edge. Second, the size of the separation bubble in the flap kink differs considerably. This relates to the generally lower flow velocity on the pressure side for the low angle of attack.

Figure 4 illustrates the turbulence kinetic energy calculated by the RANS simulation. As the streamlines before, it shows well the region of partially separated flow on the flap. Furthermore, the general turbulence levels on the airfoil's suction side is higher for the high angle of attack. This relates to the stronger acceleration and deceleration that can also be seen in figure 3 .

\section{II.B. Aeroacoustic Source Simulation}

In the following paragraphs the numerical approaches to model the relevant acoustic sources are presented. Thereby, two general source mechanisms are considered, both based on the fRPM method that is based on stochastic turbulence reconstruction by filtering. ${ }^{9}$ On the one hand the jet mixing noise is based on the model formulated by Tam and Auriault. ${ }^{11}$ On the other hand, an eddy relaxation source term formulated by Ewert et al. ${ }^{12}$ is used to account for turbulence induced noise interacting with the geometry of the airfoil. Both methods provide source terms that are directly used with the Linearized Euler Equations (LEE).

\section{II.B.1. Jet Mixing Noise}

The jet mixing noise source, that is related to turbulence-turbulence interaction, is modeled by a time-domain formulation of the jet-noise model by Tam and Auriault ${ }^{11}$ where the sources gained from the turbulence reconstruction are directly linked to the model source term. ${ }^{8}$ It was shown that this method gives good results considering round jets. ${ }^{13}$ Furthermore, Ahuja et al. ${ }^{14}$ showed, that the Tam and Auriault model is also applicable to high aspect ratio jets that are to be considered in the case of circulation control airfoils. Hence it is assumed that the time-domain formulation of the Tam and Auriault model is also valid for the present case.

\section{II.B.2. Turbulence-Geometry Interaction Noise}

As described in section I, the jet mixing noise is only one acoustic source mechanism amongst others. Therefore, further simulations are carried out concentrating on interaction noise of turbulence with the 


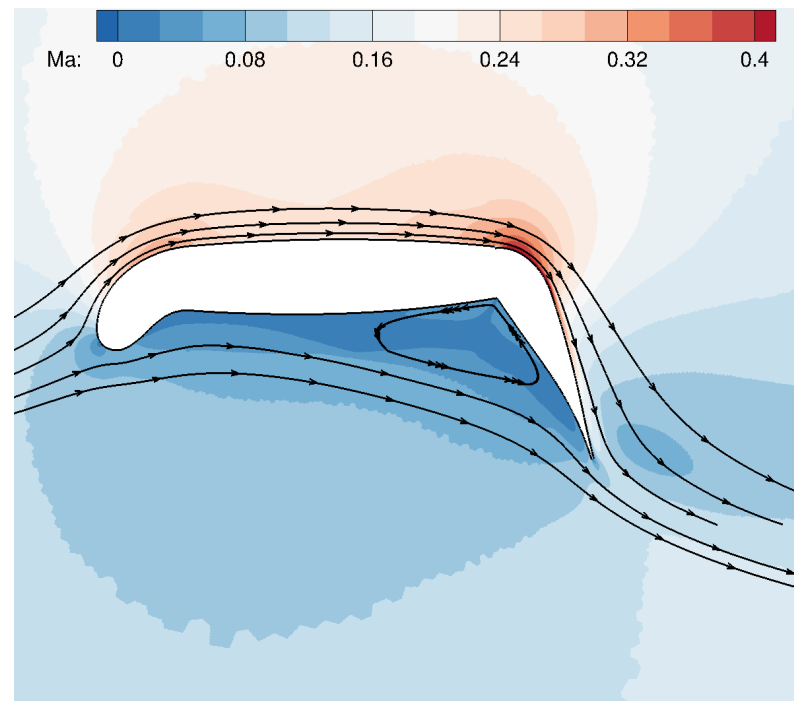

(a) Setup 1: $c_{\text {flap }}=30 \% \cdot c, \alpha=0^{\circ}, M a_{\text {jet }}=0.9\left(c_{\mu}=0.062\right)$

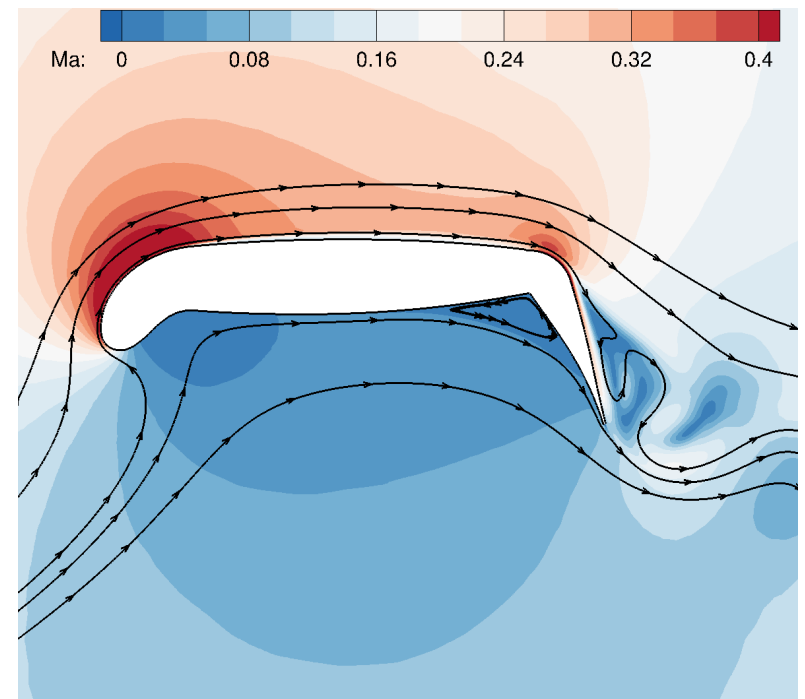

(b) Setup 2: $c_{\text {flap }}=25 \% \cdot c, \alpha=15^{\circ}, M a_{\text {jet }}=1.1\left(c_{\mu}=\right.$ $0.058)$

Figure 3: Mean flow around airfoil with local Mach number for the two setups $\left(R e=1 \cdot 10^{6}, M a_{\infty}=0.15\right)$.

airfoil. With the use of the fRPM method individual source locations can be investigated by placing the reconstruction domain in the respective region. Here, three areas are considered specifically corresponding to those listed in section I:

- the trailing edge of the flap (red box in figure 2)

- the suction side of the flap with strongly curved flow and jet slot (green box in figure 2)

- the kink with detached flow on the pressure side of the flap (blue box in figure 2)

Similar noise sources that are based on turbulence-geometry interaction, such as airfoil trailing-edge noise or high-lift slat noise have already been successfully approached with different source formulations. ${ }^{8,9,15-17}$ Therewith, the fluctuating vorticity is implemented a a deterministic source term on the right-hand side of the Acoustiuc Perturbation Equations (APE). ${ }^{18}$ However, this method is not directly applicable to the acoustic simulations of the circulation-controlled airfoil. Observation showed that the strong gradients in the blowing jet trigger local instabilities, that are not able to propagate with the mean flow by the formulation of the APE and thus keep growing. Therefore, a different source formulation is required, that goes together with solving the Linearized Euler Equations that allow this propagation. Figure 5 illustrates an approach that was formulated by Ewert et al. ${ }^{12}$ Thereby, the turbulence reconstruction method fRPM is used to add a $4 \mathrm{D}$ spatial and temporal vorticity source term $S_{v, i}$ to the momentum equation of the LEE by comparing the target vorticity $\omega_{i}^{t}$ from the reconstruction to the vorticity calculated in the CAA domain $\omega_{i}^{\prime}$ (cf. eq. 1). The coupling is then realized by the relaxation parameter $\sigma$ which is of order of magnitude of $10^{-4}$. This forcing source formulation was already tested and used for different applications, such as jet noise predictions ${ }^{19}$ jet-wing interaction. ${ }^{20}$

$$
S_{v, i}=-\frac{\partial}{\partial x_{i}} \times\left[\sigma\left(\omega_{i}^{\prime}-\omega_{i}^{t}\right)\right]
$$




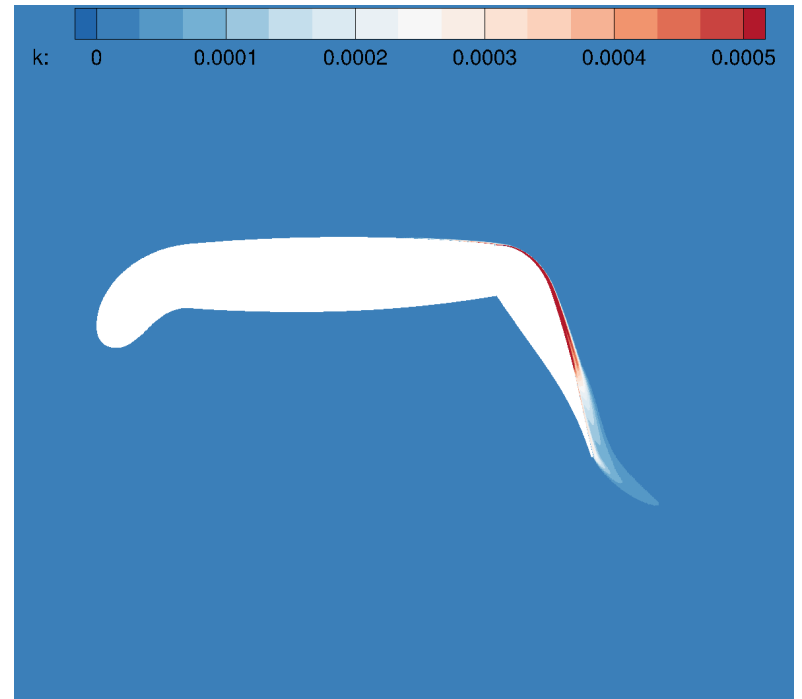

(a) Setup 1: $c_{\text {flap }}=30 \% \cdot c, \alpha=0^{\circ}, M a_{j e t}=0.9\left(c_{\mu}=0.062\right)$

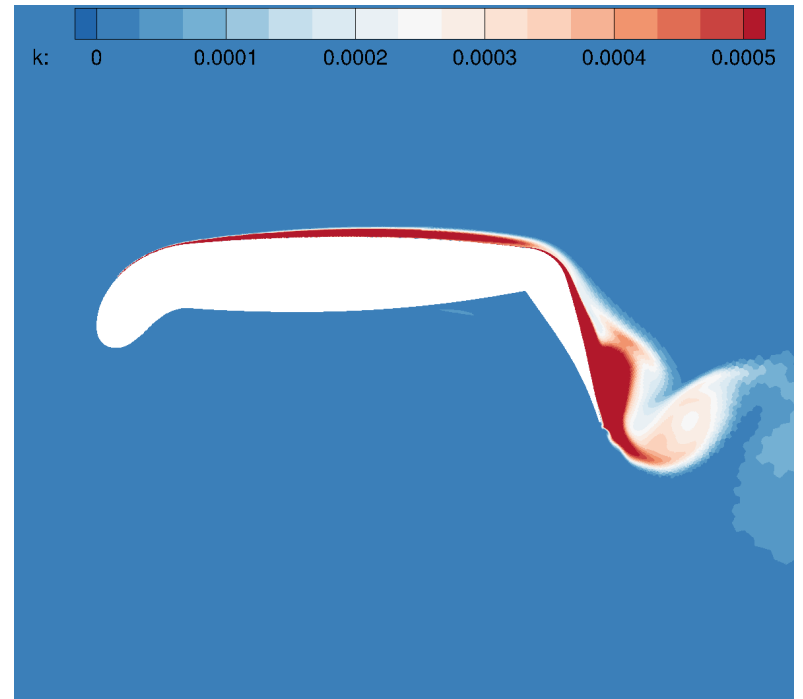

(b) Setup 2: $c_{\text {flap }}=25 \% \cdot c, \alpha=15^{\circ}, M a_{\text {jet }}=1.1\left(c_{\mu}=\right.$ 0.058)

Figure 4: Mean flow around airfoil with turbulent kinetic energy for the two setups $\left(\operatorname{Re}=1 \cdot 10^{6}, M a_{\infty}=\right.$ $0.15)$.

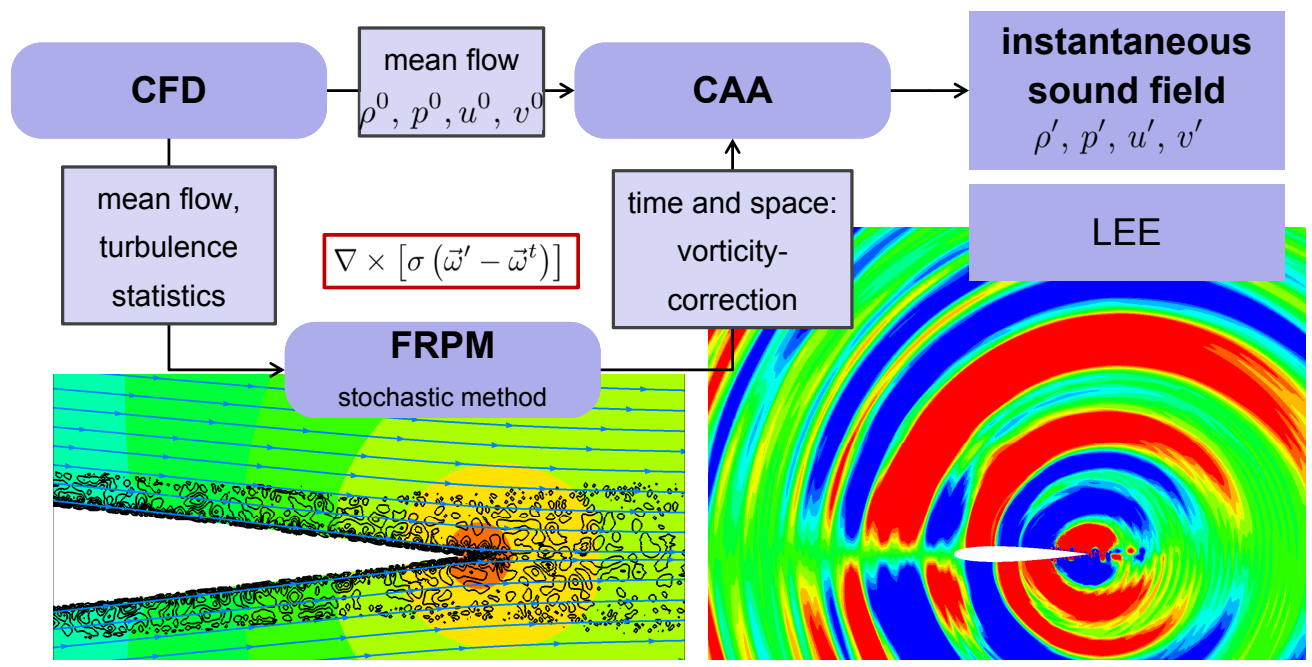

Figure 5: Flowchart of eddy relaxation source term of fRPM (fast Random Particle Mesh) turbulence reconstruction method in use with CAA code PIANO. ${ }^{21}$ 


\section{Experimental Setup}

Measurements were performed in the Acoustic Wind Tunnel Braunschweig (AWB) at DLR Braunschweig, Germany. This wind tunnel has an open jet test section and a maximum speed of up to $65 \frac{\mathrm{m}}{\mathrm{s}}$ with a turbulence intensity of up to $0.3 \%$. It is designed to conduct acoustic measurements. The test section is covered by anechoic wedge foams to reduce wall reflections.

The rectangular nozzle of the wind tunnel is $0.8 \mathrm{~m}$ wide and $1.2 \mathrm{~m}$ high. Acoustically lined side walls were mounted to the nozzle to hold the airfoil model. The airfoil model that was used in this study was previously installed in the MUB closed test section wind tunnel of the Institute for Fluid Mechanics at the Technical University Braunschweig for aerodynamic measurements. The cross-sectional area of the MUB is larger than the nozzle area of the AWB. The model, based on the DLR F16 airfoil, has a clean chord length of $c=0.3 \mathrm{~m}$ and a span width of $b=1.3 \mathrm{~m}$. With droop nose and a $30 \% \cdot c$ flap deflected at $65^{\circ}$, the geometric chord length is $0.23 \mathrm{~m}$.

The circulation control via high-speed jet is realized by a high-aspect ratio slit nozzle on the suction side ahead of the flap segment. The height of the slit $h_{\text {slit }}$ equals $0.2 \mathrm{~mm}$ and the slit width extends the full span width of the model. The high-speed jet was fed through the sides of the model from a compressor outside of the test section.

The model was installed horizontally between the side walls, so blowing was only needed for the span width of $b_{\text {slit }} 0.8 \mathrm{~m}$. Outside of the side walls, the jet would produce spurious noise that can contaminate the measurements. In the first attempt, the jet slit in the excess portions of the model were covered by aluminum tape, which didn't resist the high pressure. So the excess portion of the model was covered in foam to reduce the acoustic impact, making airflow leakage unavoidable.

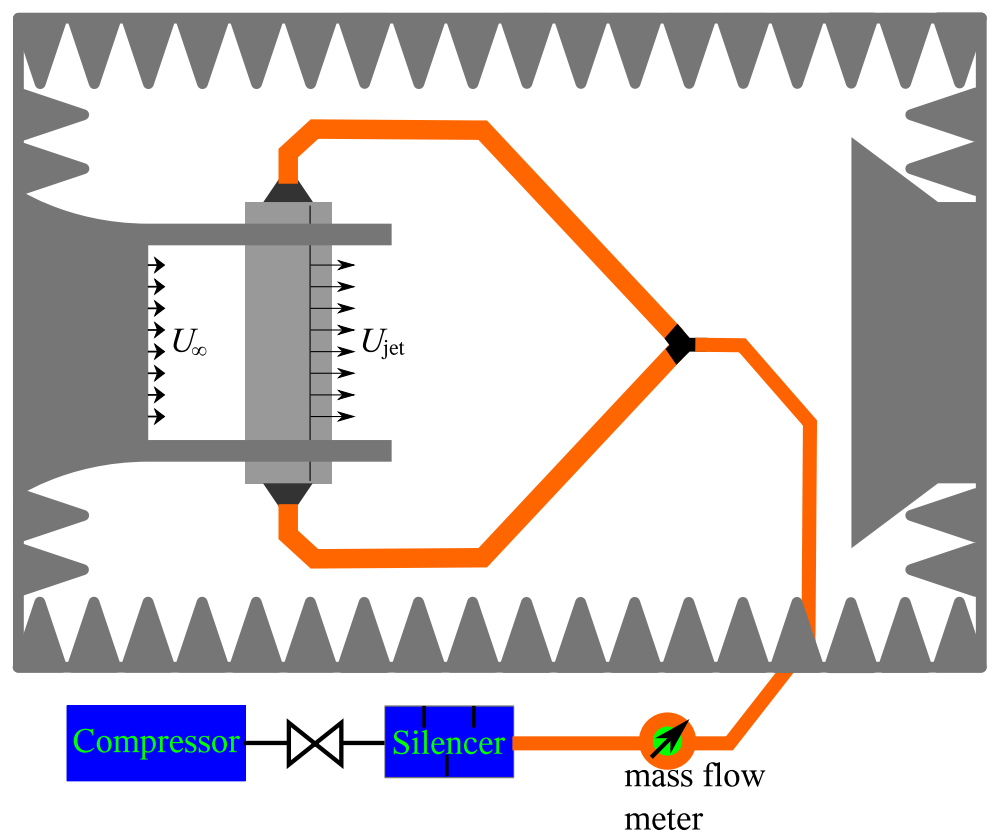

Figure 6: Experimental setup of the high speed jet for airfoil circulation control. Only the test section of the AWB is shown.

\section{III.A. Correction of $c_{\mu}$ for Excess Jet}

A mass flow meter monitors the air flow going into the airfoil model, from which the jet momentum coefficient $c_{\mu, \text { meas }}$ is calculated. This quantity has to be corrected for the leakage through the excess jet slit. The measured $c_{\mu, \text { meas }}$ is defined as

$$
c_{\mu, \text { meas }}=\frac{\dot{m} U_{\text {jet }}}{0.5 \rho_{\infty} U_{\infty}^{2} A_{\text {ref }}}
$$

where $\dot{m}$ is the mass flow as measured by the mass flow meter, $A_{r e f}=0.3 \mathrm{~m} \times 0.8 \mathrm{~m}$ is the effective wing area of the model inside the test section and $U_{j e t}$ is the jet velocity that is defined by interpolating the static 


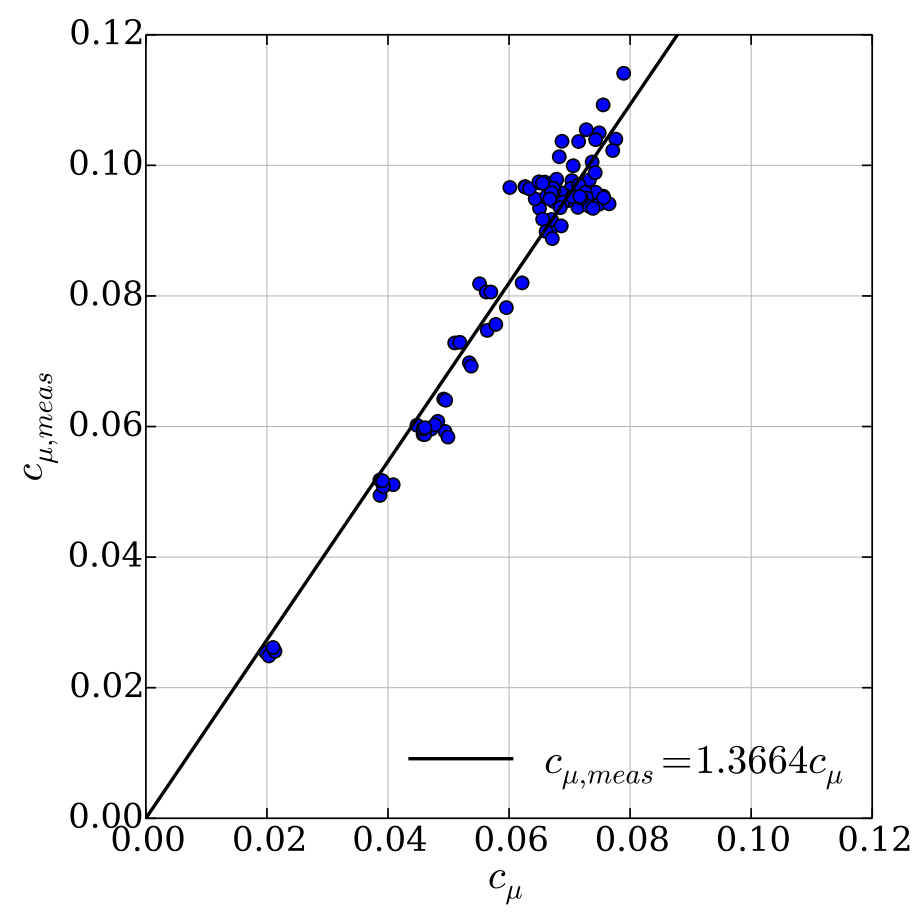

Figure 7: Linear regression between $c_{\mu, \text { meas }}$ and corrected $c_{\mu}$.

Table 2: Correction table for $c_{\mu}$.

\begin{tabular}{rr}
$c_{\mu}$, meas & $c_{\mu}$ \\
\hline 0.060 & 0.044 \\
0.090 & 0.066 \\
0.095 & 0.069 \\
0.100 & 0.073 \\
\hline
\end{tabular}

pressure located at positions upstream and downstream of the jet slit. The calculation of $U_{j e t}$ is defined as

$$
U_{\text {jet }}=0.96\left[\frac{2 \gamma R_{s} T_{\text {plenum }}}{\gamma-1}\left(1-\frac{p_{\text {jet }}}{p_{\text {plenum }}}\right)^{\frac{\gamma-1}{\gamma}}\right]^{1 / 2}
$$

where $T_{\text {plenum }}$ and $p_{\text {plenum }}$ are the temperature and static pressure measured in the plenum, $\gamma=1.4$ is the adiabatic index, and $R_{s}=287.058 \frac{\mathrm{J}}{\mathrm{kg} \mathrm{K}}$ is the specific gas constant.

The measured mass flow $\dot{m}$ is the sum of mass flow used for circulation control and mass flow from the excess parts. The mass flow of the jet exiting the reduced span slit is defined as $\dot{m}=\rho_{\text {jet }} U_{\text {jet }} h_{\text {slit }} b_{\text {slit }}$, enabling to rewrite equation (2) to

$$
c_{\mu}=\frac{\rho_{\text {jet }} U_{\text {jet }}^{2} b_{\text {slit }} h_{\text {slit }}}{0.5 \rho_{\infty} U_{\infty}^{2} A_{\text {ref }}}
$$

The jet momentum coefficient used for circulation control is therefore a function of $h_{\text {slit }}, b_{\text {slit }}$, and $\rho_{\text {jet }}$ compensated for compressibility by

$$
\rho_{j e t}=\rho_{\infty}\left(\frac{T_{j e t}}{T_{\infty}}\right)^{\frac{1}{\gamma-1}}
$$

The relation between $c_{\mu, \text { meas }}$ and $c_{\mu}$ in Eq. (6) is formulated by drawing a linear regression of the available data as shown in figure 7

$$
c_{\mu, \text { meas }}=1.3664 c_{\mu}
$$

$$
8 \text { of } 23
$$


The measured and corrected values are shown in table 2. Boundary layer reattachment on the flap segment was observed in the AWB when $c_{\mu}=0.066$. This value was reported in the MUB as $c_{\mu}=0.062$, which is an acceptable variation of $6.4 \%$.

\section{III.B. Aerodynamic measurements}

The model is equipped with 63 static pressure ports to measure the pressure coefficients of the model at $\alpha_{A W B}=6^{\circ}$ and $11^{\circ}$ that are equivalent to $\alpha_{M U B}=0^{\circ}$ and $5^{\circ}$, respectively. These angles were selected to have comparable pressure coefficients with measurements that were performed in the MUB. The comparison is judged good when the pressure coefficients on the flap segment from AWB and MUB overlap each other. The two sets of pressure coefficients are shown in figure 8 .

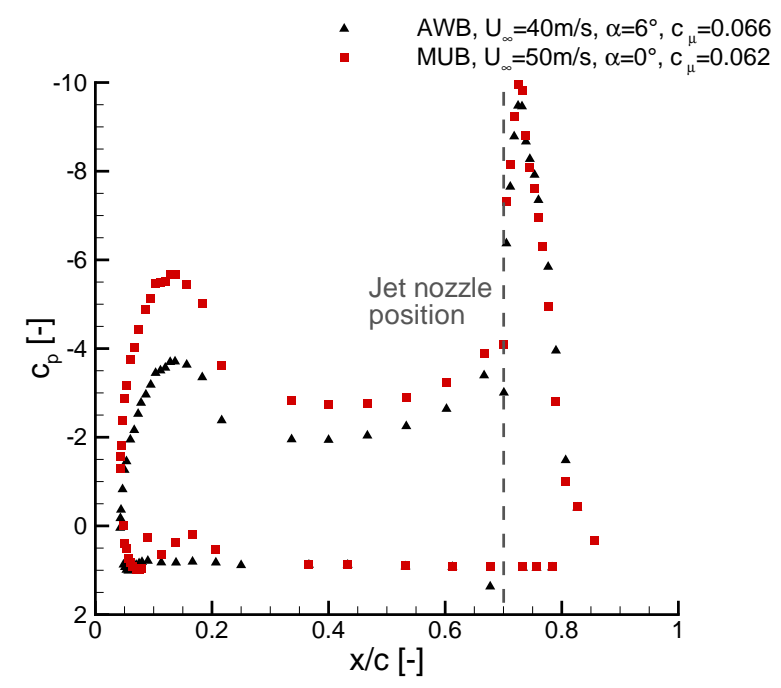

(a)

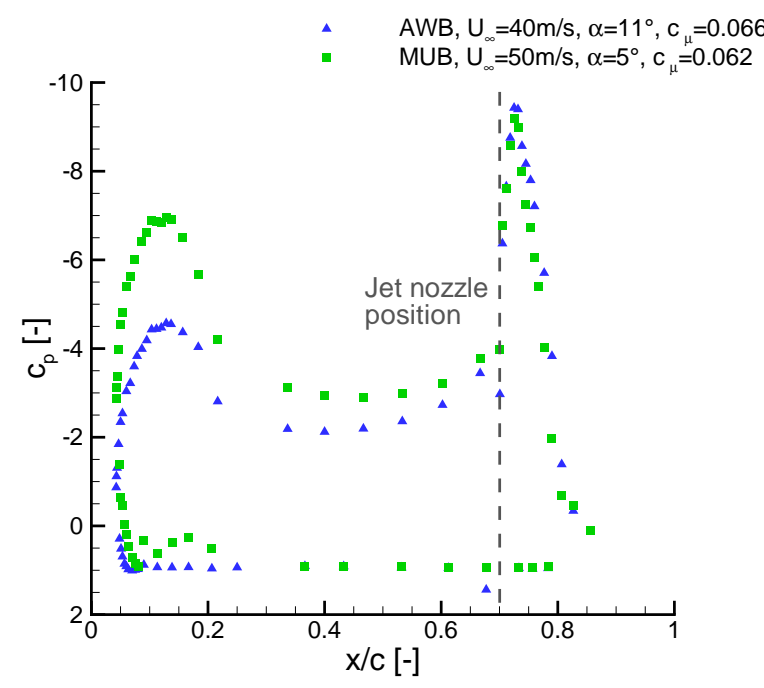

(b)

Figure 8: Pressure coefficient for $c_{\mu}$ of boundary layer reattachment for different angles of attack.

\section{III.C. Acoustic Measurements}

Three acoustic measurement systems were used in this study. Free-field microphones, a large aperture microphone array with 96 microphones, and a small aperture microphone array with 48 microphones. The three measurements were done separately, but all measurements were ensured to be statistically stationary by sampling interval longer than 10 seconds.

\section{III.C.1. Free-Field Microphones}

Two lines of microphones were arranged above and below the midspan of the airfoil. Each line consisted of 8 microphones, 1/4 inch Brüel \& Kjær Type 4136 located in both up- and downstream direction. The line microphones below the model are positioned at different heights from the floor to reduce interaction of the wind tunnel's shear layer with the microphones. Figure 9 shows the arrangement of these free-field microphones. The associated coordinates are listed in table 3. A sampling frequency of $100 \mathrm{kHz}$ was used resulting in 1,000,000 samples collected.

\section{III.C.2. Microphone Arrays}

The large aperture microphone array consisting of 96 1/2 inch Linear-X M51 microphones was positioned $0.98 \mathrm{~m}$ above the model. Post-processing uses the CLEAN-SC algorithm of Sijtsma. ${ }^{22}$ A $10 \mathrm{~mm} \times 10 \mathrm{~mm}$ post-processing grid was used to analyze the acoustic source maps. Based on the acoustic source map, the free-field sound spectrum was calculated by local area integration. The sound spectra from the free-field microphone and microphone array are compared. 
Table 3: Free-field microphone placements relative to the center of the nozzle exit plane. All measures in $\mathrm{mm}$.

\begin{tabular}{rrrl}
\hline Mic. \# & $x$ & $y$ & $z$ \\
\hline 1 & 0 & -850 & 0 \\
2 & 298 & -875 & 0 \\
3 & 498 & -892 & 0 \\
4 & 647 & -905 & 0 \\
5 & 747 & -914 & 0 \\
6 & 896 & -926 & 0 \\
7 & 1095 & -943 & 0 \\
8 & 1394 & -969 & 0 \\
9 & 7 & 983 & 8 \\
10 & 220 & 983 & 8 \\
11 & 485 & 983 & 8 \\
12 & 618 & 983 & 8 \\
13 & 734 & 983 & 8 \\
14 & 1135 & 983 & 28 \\
15 & 1383 & 983 & 28 \\
16 & 1633 & 983 & 28 \\
\hline
\end{tabular}

A small aperture array consisting of 48 1/4 inch Brüel \& Kjær Type 4954A microphones was positioned below the model at $y=-1296 \mathrm{~mm}$. To measure the directivity of the far-field noise, the array was positioned at three different downstream stations at $x=426 \mathrm{~mm}, 770 \mathrm{~mm}$ and $1205 \mathrm{~mm}$. All measurements were conducted with the center of the array positioned below the midspan of the model. The same signal postprocessing for analyzing the results from the large-aperture microphone array was applied for the smallaperture one. The sampling frequency was set to $64 \mathrm{kHz}$. The setup for both arrays is shown in figure 10.

\section{III.D. Measurement Parameters}

The measurement parameters are shown in table 4. Jet noise was measured with $U_{\infty}=0 \frac{\mathrm{m}}{\mathrm{s}}$ and $U_{\text {jet }}=$ $170,200,230$ and $260 \frac{\mathrm{m}}{\mathrm{s}}$. Baseline measurement provides the reference, where the airfoil's circulation is not controlled. Coanda measurements were performed with free stream velocities of $U_{\infty}=30,35$ and $40 \frac{\mathrm{m}}{\mathrm{s}}$. Background noise was measured with $U_{\infty}=30,35,40$ and $50 \frac{\mathrm{m}}{\mathrm{s}}$ after the airfoil model was removed from the test section.

Table 4: Measurement parameters

\begin{tabular}{lll}
\hline Parameter & Magnitude & Notes \\
\hline$U_{\infty},\left[\frac{\mathrm{m}}{\mathrm{s}}\right]$ & 0 & jet noise measurement \\
& $30,40,50$ & \\
\hline$\alpha_{A W B},\left[^{\circ}\right]$ & 6,11 & \\
\hline$c_{\mu}$ & 0 & baseline measurement \\
& $0.066,0.069,0.073$ & Coanda measurements \\
\hline
\end{tabular}




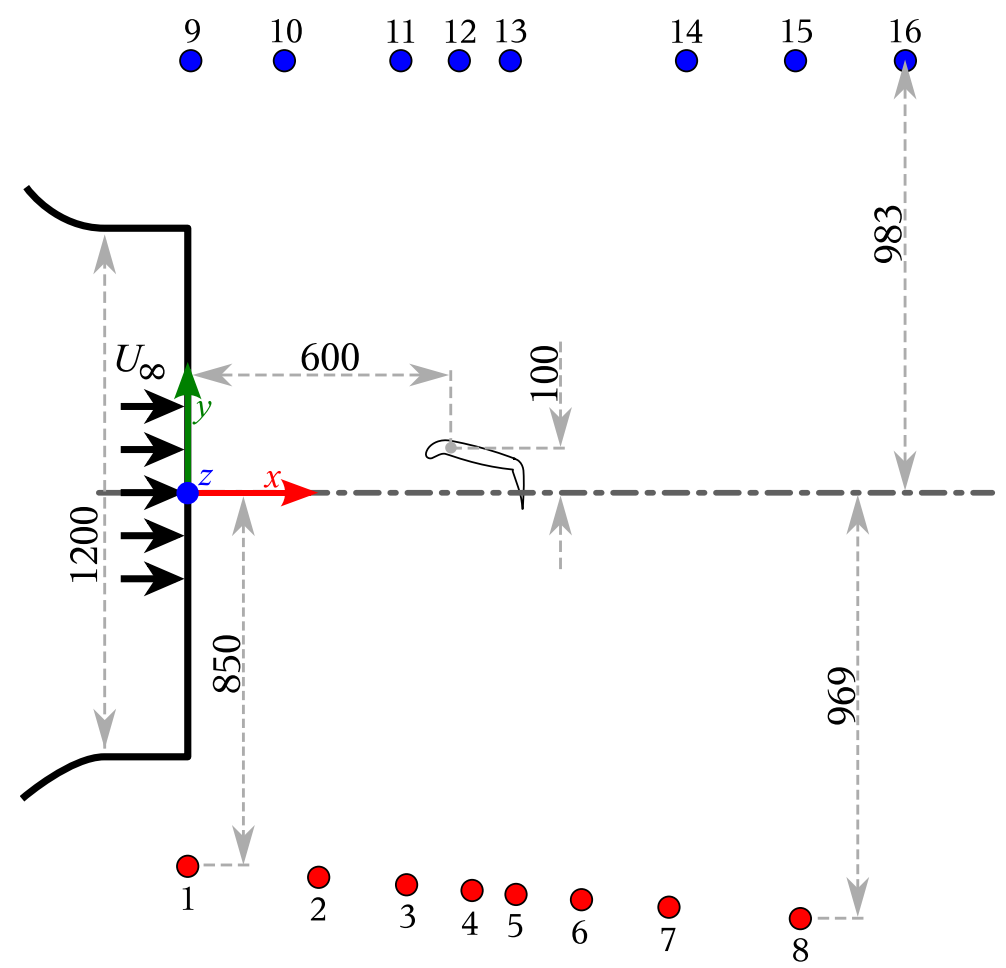

Figure 9: Far-field microphones arrangement with respect to the model installed in the AWB. 


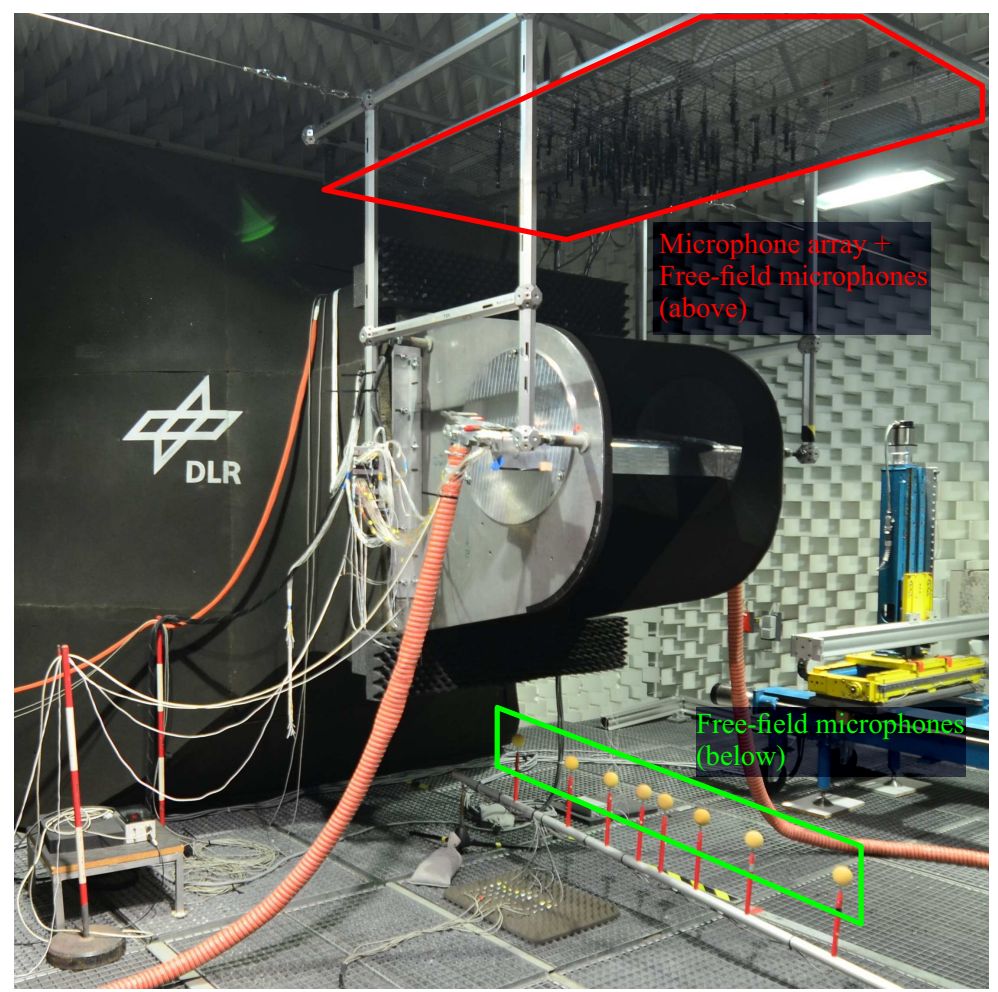

(a) Free-field microphones and microphone array above the model and freefield microphones below the model

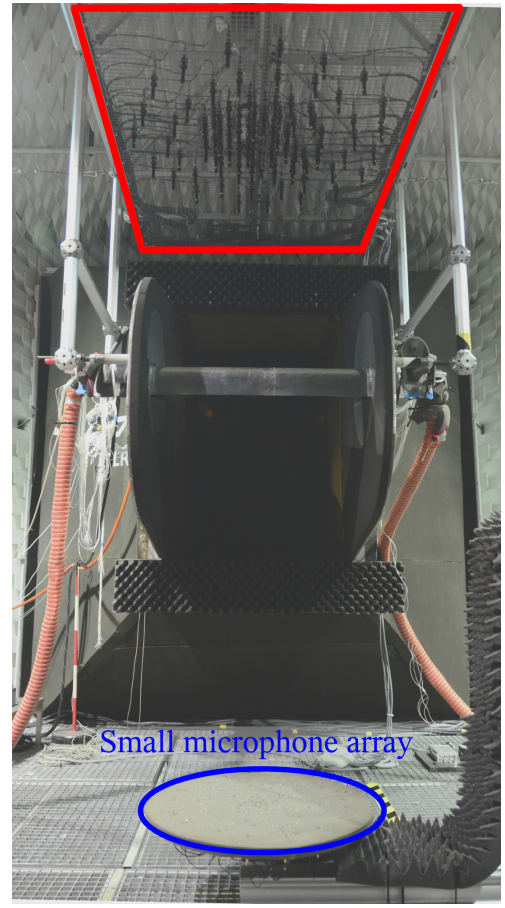

(b) Large and small aperture microphone arrays

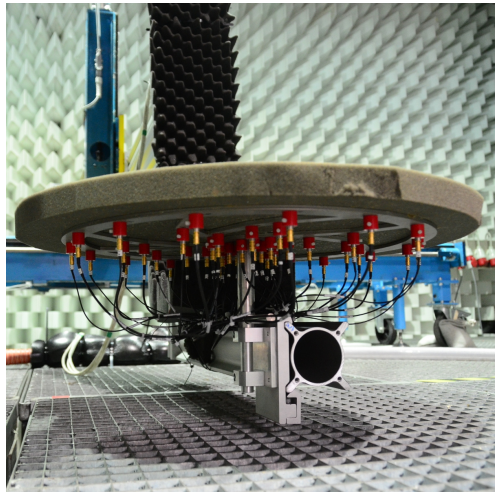

(c) Small microphone array

Figure 10: Acoustic measurements setup in the AWB. 


\section{Results}

In the following sections the results from both simulations and experiments regarding the different noise source mechanisms are presented. It has to be noted that on the numerical side for the jet mixing noise a different source model compared to the subsequent simulations is used. Therefore in this 2D setup, the absolute sound pressure levels are not directly comparable. Furthermore, the levels obtained by the simulations are not calibrated to the experimental results. Hence, only qualitative comparison between the different results are feasible.

\section{IV.A. Jet Mixing Noise}

\section{IV.A.1. Simulation}

The first considered source mechanism is the jet mixing noise, based on the model formulated by Tam and Auriault. ${ }^{11}$ Figure 11 shows a snapshot of the sound pressure field that is radiated and the directivity of the overall sound pressure level (OASPL). It can be seen that this source produces relatively high frequency noise that mainly radiates to the top. Below the airfoil a significant shielding effect can be observed, which plays a favorable role considering community noise. The directivity plot reveals the dependency of the noise on the jet velocity and the state of the mean flow. It can be seen that with the higher jet velocity the sound pressure level generally increases, which is expected. However, for radiation angles between $120^{\circ}$ and $240^{\circ}$ a different behavior is observed. This is probably an effect related to the different angle of attack between the two setups, that causes a tilt of the radiation pattern by the changed mean flow.

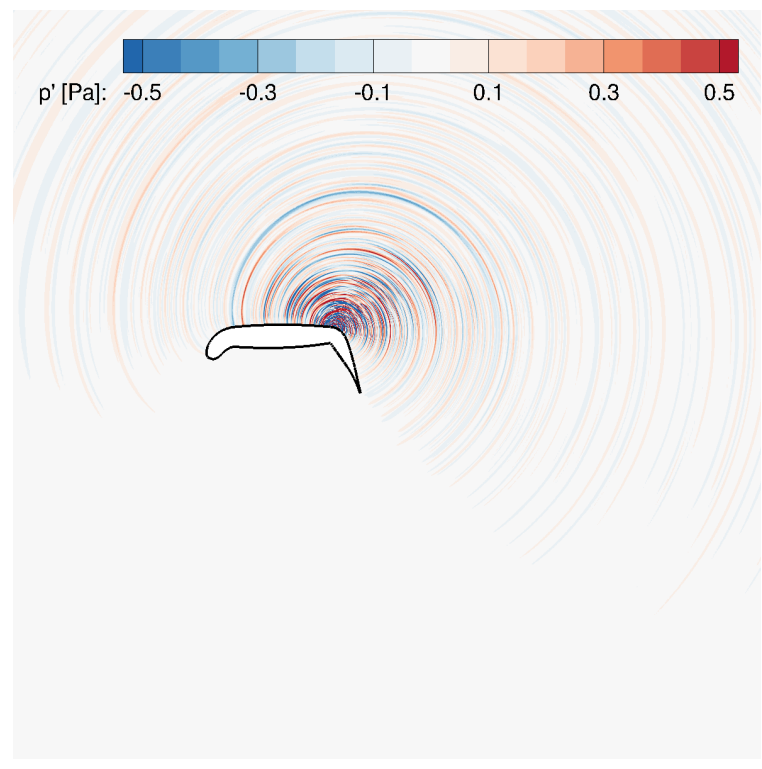

(a) Snapshot of the jet induced sound pressure field (setup 1)

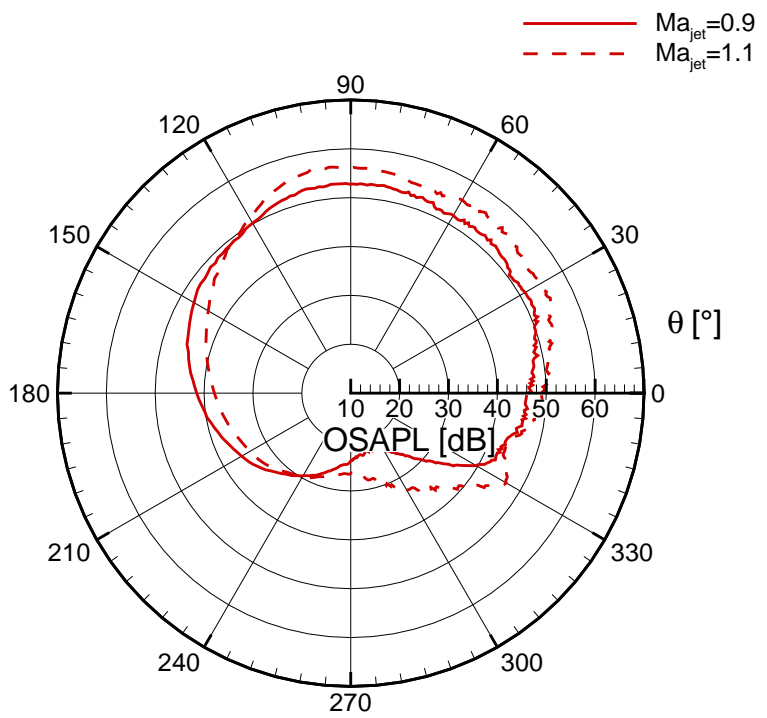

(b) Directivity of the OASPL $(300 \mathrm{~Hz}-30 \mathrm{kHz})$ at $1.5 \cdot c$ from the trailing edge for different jet velocities: $M a_{\text {jet }}=0.9$ (setup 1, solid line), $M a_{j e t}=1.1$ (setup 2, dashed line)

Figure 11: Simulation results for the jet noise source.

An exemplary spectrum of the jet mixing noise for a radiation angle of $\theta=45^{\circ}$ is shown in figure 12 . It reveals, that for both jet velocities the spectra rise up to the resolution limit of the mesh. This is according to the very narrow jet outlet and the high velocity. Furthermore, the faster jet comes with higher sound pressure levels for all frequencies.

\section{IV.A.2. Experiment}

Jet mixing noise was investigated in the experiments by turning the compressor on while the tunnel was not running. Acquisition of the radiated noise was possible using the free-field microphones as well as the large aperture microphone array positioned above the wing (see figure 10). The resulting spectra for different values of the jet velocity, $U_{j e t}$, are plotted in figure 13 . In figure $13 \mathrm{a}$, a comparison is made 


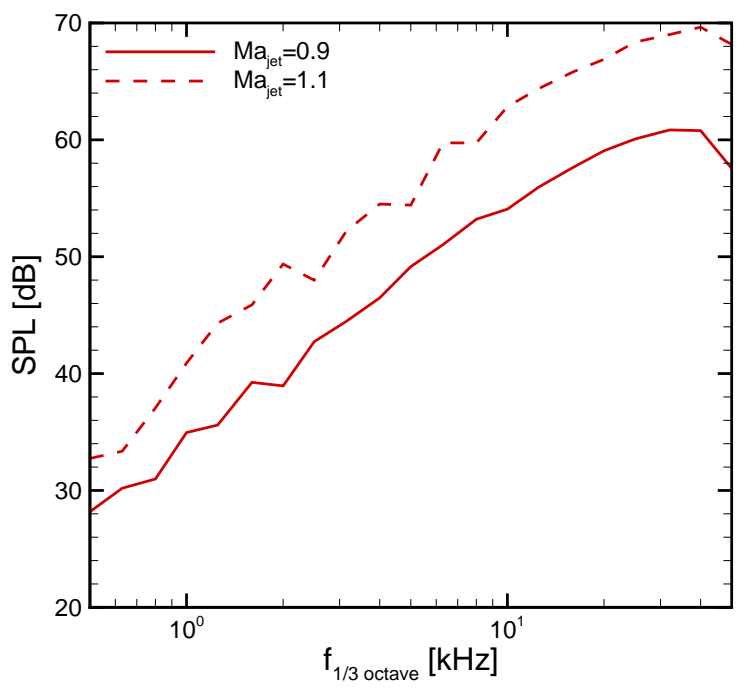

Figure 12: Calculated spectra for the jet noise source at an angular position of $\theta=45^{\circ}$ at a distance of $1.5 \cdot c$ from the trailing edge.

between the microphone array results and the far field measurement at microphone 12 (e.g. figure 9). Both measurement techniques are found to capture the radiated noise in principle. Of the two techniques, the free-field microphones deliver data over a more important frequency range, nominally at high frequencies. The advantage of the microphone array lies in its implicit focusing abilities which will be needed later on when dealing with co-flow situations.

In figures $13 \mathrm{~b}, \mathrm{c}$ two different scalings are applied to the data. Here, we assume $p_{r m s}^{2} \propto U_{\text {jet }}^{n}$ with $n=6.5$ in figure 13b and $n=8.0$ in figure 13c. These power exponents are picked because they provide the best collapse of the spectra. The dimensionless frequency axis is calculated using an arbitrary length scale of $1 \mathrm{~m}$ and $U_{j e t}$. The results of figure 13b suggest an turbulence-geometry interaction noise source mechanism, mostly important at lower frequencies, which could be originating at the slit lip as proposed by Williams. ${ }^{23} \mathrm{He}$ suggested that the jet exit geometry could affect the noise radiation by an additional component radiating as a fluctuating force dipole source with $p_{r m s}^{2} \propto U_{\text {jet }}^{6}$. Furthermore, Seiner et al. ${ }^{24}$ assume $p_{r m s}^{2} \propto U_{j e t}^{7}$, due to a mixing of turbulent jet mixing noise and lip noise, to explain the results of their investigations. In figure 13c, the data scaling indicates that jet mixing noise is dominant at higher frequencies, which is expected due to the small geometric scale of the model slit. However, the relatively low-frequency noise might also originate from the bending of the high-speed jet around the flap. This so-called curvature noise will be discussed further in section IV.C.

Measurements with free stream velocity were also run using a small aperture microphone array traversed on the fly-over line below the wing (see figure 14a). The corresponding results are plotted in figure 14b. As the microphone is moved upstream, the jet mixing noise contribution is found to diminish due to shielding from the wing, corroborating the simulations. However, the low frequency part remains mostly constant. Therefore, it is probably related to other source mechanisms that will be discussed in the following. 


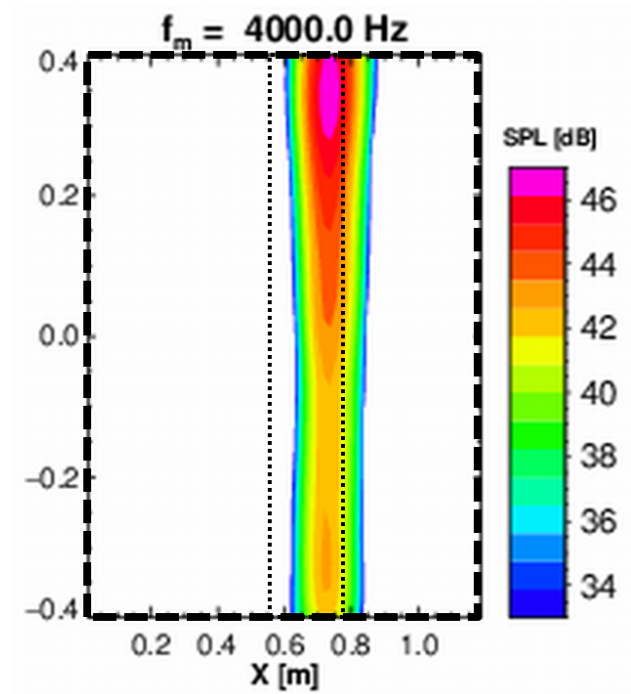

(a) Source map $f_{1 / 3}$ octave $=4 \mathrm{kHz}$. Scan grid: long-dash rectangle, model: dotted rectangle

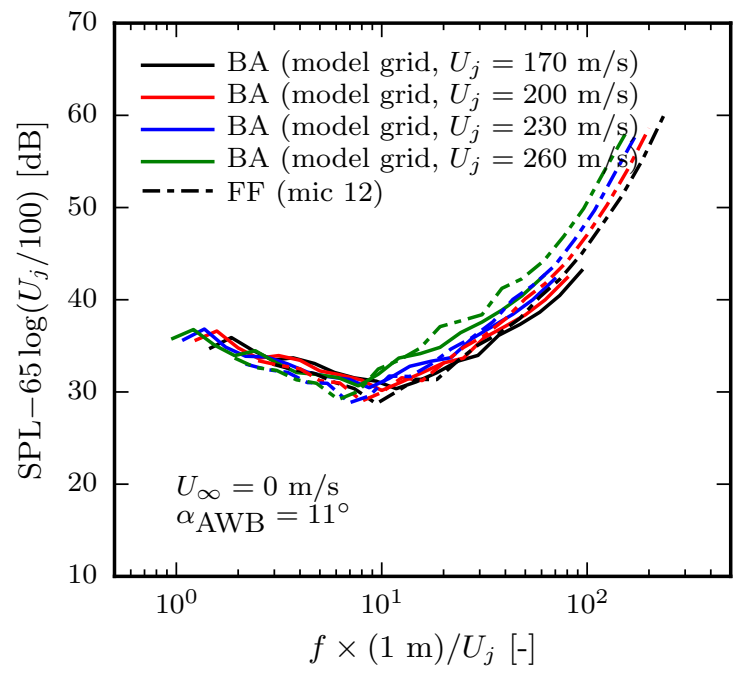

(c) Scaled data, $n=6.5$

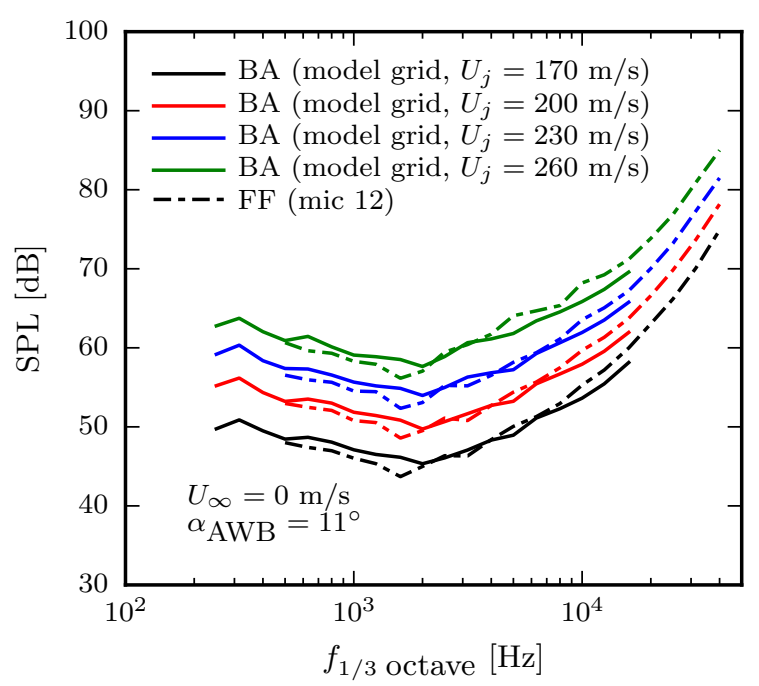

(b) Measured data

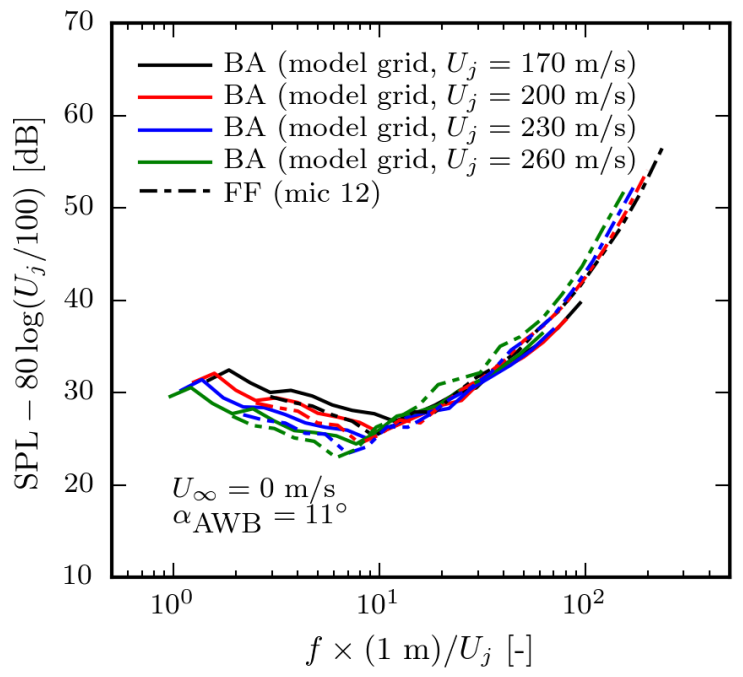

(d) Scaled data, $n=8.0$

Figure 13: Jet only, scaling assuming $p_{r m s}^{2} \propto U_{j e t}^{n}, U_{\infty}=0 \frac{\mathrm{m}}{\mathrm{s}}, \alpha_{A W B}=11^{\circ}, \mathrm{BA}=$ large aperture microphone array, $\mathrm{FF}=$ free-field microphone. 


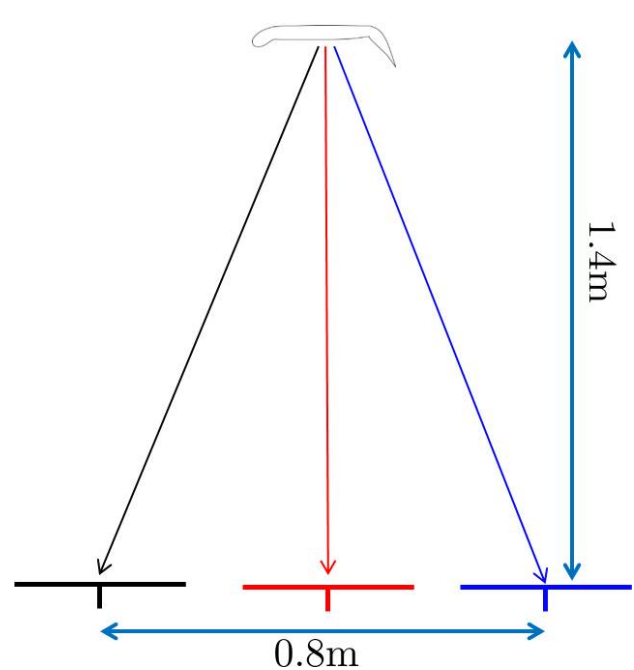

(a) Microphone array positions

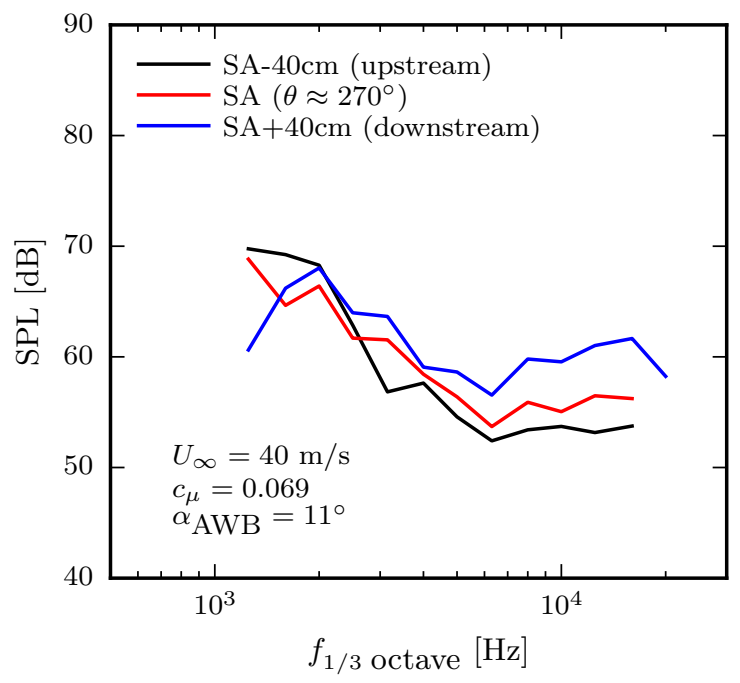

(b) Microphone array results

Figure 14: Fly-over line noise radiation directivity. $U_{\infty}=40 \frac{\mathrm{m}}{\mathrm{s}}, \alpha_{A W B}=11^{\circ}, c_{\mu}=0.069$. Small aperture array data. 


\section{IV.B. Trailing Edge Noise}

\section{IV.B.1. Simulation}

By avoiding the classic high lift noise from a slat, the noise generated at the trailing edge of the flap is supposed to become a significant acoustic source. The noise radiated from this specific source is illustrated in figure 15 with the source region marked by the red box. It can be seen that no classic cardioid directivity pattern as expected for trailing edge noise is formed. This is most probably due to the highly curved mean flow around the airfoil that leads to a deformation of the emitted sound waves. Comparing the directivity pattern at different angles of attack it shows that for $\alpha=15^{\circ}$, the main radiation direction is to the bottom and front, whereas for $\alpha=0^{\circ}$ a more uniform pattern is formed. This indicates that convective amplification may play an important role. Another possible influence on the directivity are reflections of the sound coming from the trailing edge on the lower side of the airfoil. However, these are not clearly visible in the snapshot of the acoustic field. It is supposed, that reflections are limited due to refraction of the sound waves at the airfoil's boundary layer.

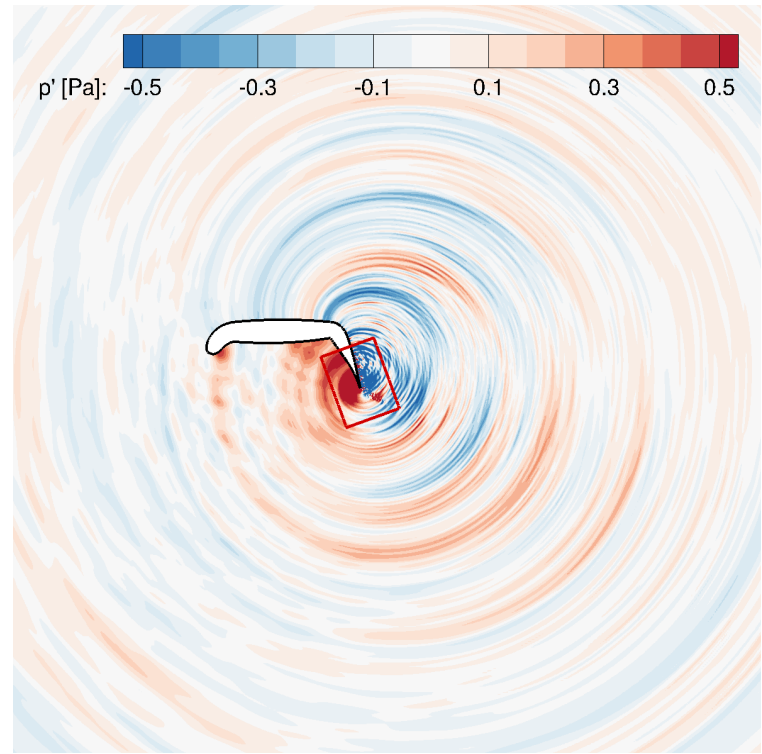

(a) Snapshot of the flap trailing edge noise sound pressure field with fRPM source region (setup 1)

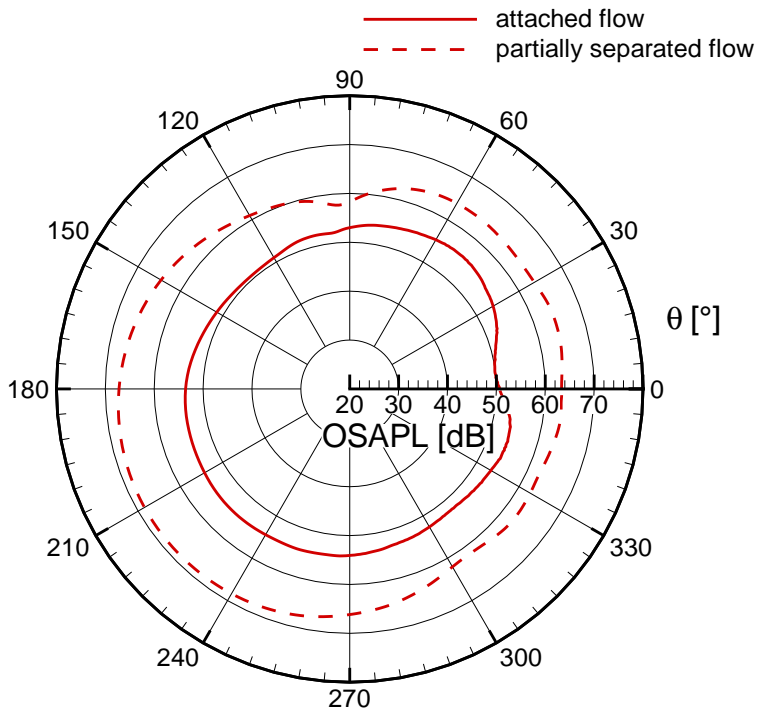

(b) Directivity of the OASPL $(300 \mathrm{~Hz}-30 \mathrm{kHz})$ at $1.5 \cdot c$ from the trailing edge for attached (setup 1, solid line) and separated flow (setup 2, dashed line)

Figure 15: Simulation results for the trailing edge noise source.

Comparing the levels of the sound radiated from the trailing edge for the two different mean flows, it shows that they rise with the angle of attack. This relates to the already presented higher turbulence kinetic energy inside the boundary layer (cf. section II.A). Furthermore, for $\alpha=15^{\circ}$, both the attached flow of the wall jet and the separated flow can contribute to the trailing edge noise, while for $\alpha=0^{\circ}$ only the attached flow remains. This should also be visible in the sound spectra, as the separated flow is expected to produce mostly low-frequency noise. To assess this issue, figure 16 shows the recorded spectra at two different radiation angles $\left(\theta=45^{\circ}\right.$ and $\left.\theta=270^{\circ}\right)$. It can be clearly seen, that the case with flow separation produces much more low- to mid-frequency sound, which conforms with the expectations from earlier measurements. ${ }^{5}$

Furthermore, for frequencies above about $8 \mathrm{kHz}$ the difference vanishes. This nicely shows the importance of getting the right flow when comparing simulation results to measurements on the one side and the acoustic impact of aerodynamically acceptable partial flow separation on the other side. This second aspect clearly needs to be considered in designing a low-noise high-lift system.

Another interesting aspect in analyzing trailing edge noise is to evaluate the weighting of the sources from the airfoil's suction side and pressure side separately. This is done by placing the respective turbulence reconstruction domains. Figure 17 shows the directivity of the radiated overall sound pressure level for the separated source regions. It can be seen that the sound produced from the pressure side turbulence is a lot weaker than from the suction side. This agrees with the lower turbulent energy on the pressure side due to 


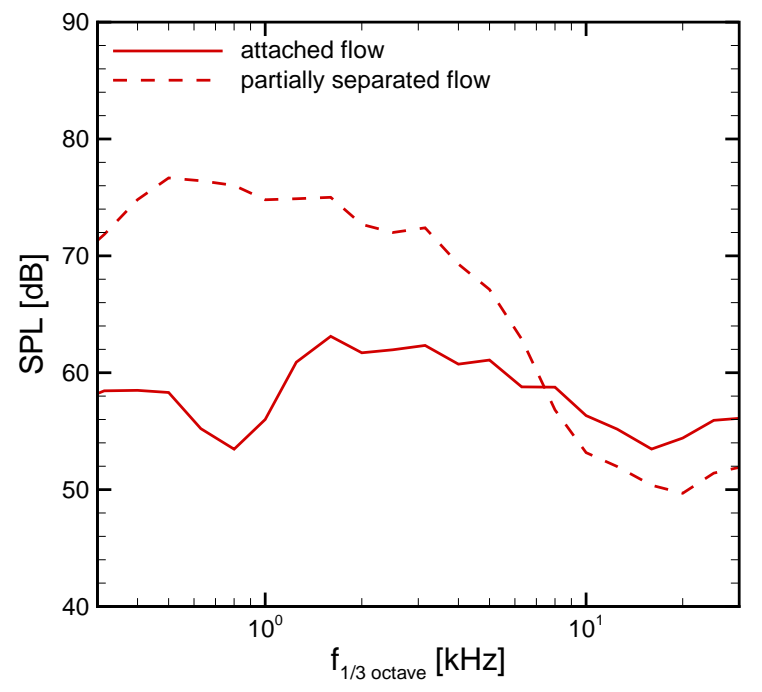

(a) Spectra recorded at an angular position of $\theta=45^{\circ}$

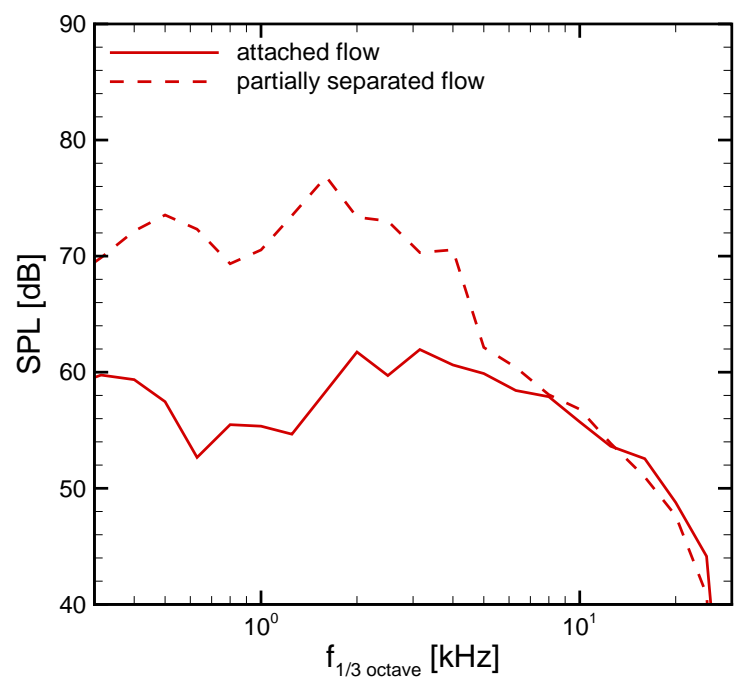

(b) Spectra recorded at an angular position of $\theta=270^{\circ}$

Figure 16: Calculated spectra for the trailing edge noise source at a distance of $1.5 \cdot c$ from the trailing edge (setup 1: solid lines, setup 2: dashed lines).

the low near wall velocity and the lack of the high-speed jet.

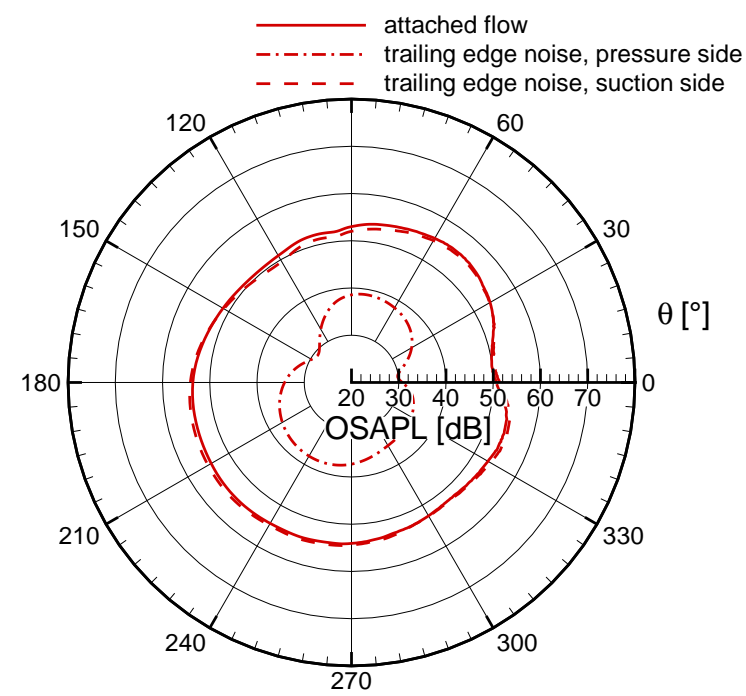

Figure 17: Directivity of the OASPL $(300 \mathrm{~Hz}-30 \mathrm{kHz})$ at $1.5 \cdot c$ from the trailing edge of the trailing edge noise split into sources on the airfoils pressure side (dash-dotted) and suction side (dashed) for setup 1 (attached flow).

\section{IV.B.2. Experiment}

Although the simulations identify the trailing edge as an important noise generator, it was not clear if it could be isolated experimentally. In the experiment, the quantification of the trailing edge noise contribution was done using the large aperture array (e.g. figure 10). The free-field microphones cannot isolate this noise source because of its low level compared to the background noise and even with the array, other source mechanisms might mask it. The approach here was to show, whether potentially noise reducing treatments on the wing can have an influence in the measured noise. If that was the case it would show that single source mechanisms were dominant. 
Considering potential noise reducing treatments, four different configurations were investigated acting on the trailing edge noise and the separation noise in the flap kink:

- reference configuration

- reference + trailing edge brush add-on

- reference + kink fairing add-on

- reference + trailing edge brush add-on + kink fairing add-on

The results of these tests are plotted in figure 18. The solid and dashed black curves correspond to the noise radiation from the reference configuration as measured using the microphone array and free-field microphone 12. In the microphone array data processing the whole of the wing is considered (i.e. region shown by the black dashed rectangle in figure 18a, including the side plate junction region). As already discussed, the agreement between the results from both measurement techniques is excellent. However, they provide a global measure of the noise generated over the complete wing. In the present case, the reduction of trailing edge noise can only be shown by reducing of the area of interest in the microphone array processing (i.e. region shown by the red dashed rectangle in figure 18a). This way, contaminations by spurious noise are successfully reduced.

From the results of figure 18b, the only beneficial treatment is the trailing edge brush, which brings 2 to $5 \mathrm{~dB}$ noise reduction in the range 1.6 to $10 \mathrm{kHz}$. On the one side, this shows, that trailing edge noise is one of the dominant noise sources and that it can be reduced through the application of known noise mitigation treatments. On the other side, it seems that the flap kink is no noise source region that can be influenced by the fairing. This aspect will be discussed later.

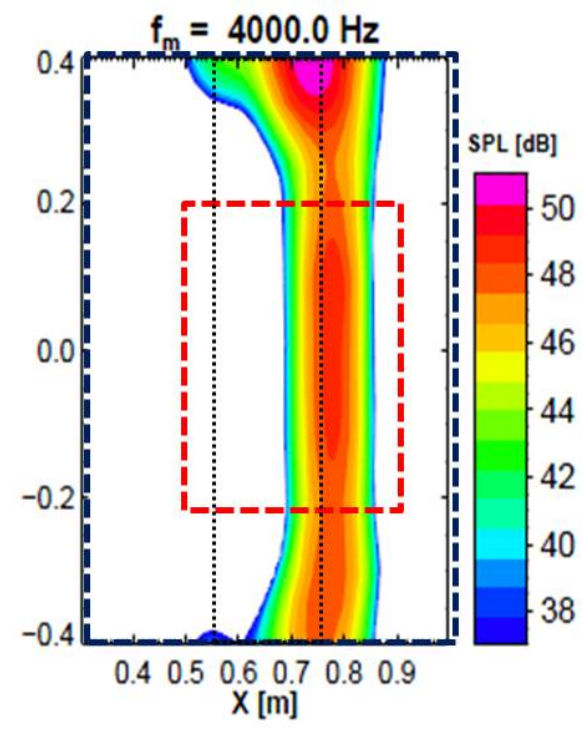

(a) Source map $f_{1 / 3}$ octave $=4 \mathrm{kHz}$. Whole scan grid: black long-dash rectangle, reduced scan grid: red long-dash rectangle, model: dotted rectangle

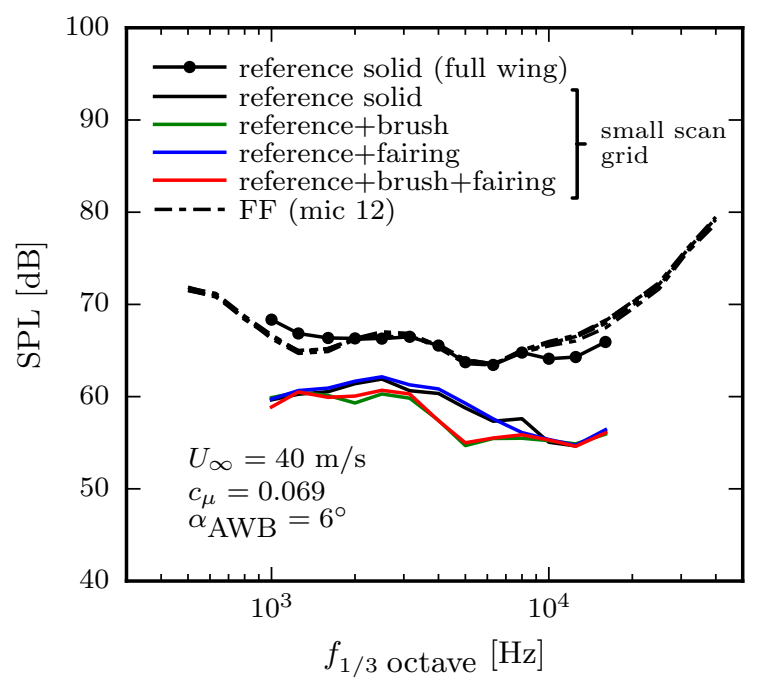

(b) Measured spectra

Figure 18: Trailing edge noise reduction, $U_{\infty}=40 \frac{\mathrm{m}}{\mathrm{s}}, \alpha_{A W B}=6^{\circ}, c_{\mu}=0.069$

\section{IV.C. Curvature Noise}

A second potential noise source that is based on the interaction of turbulence with the airfoil's geometry is the so-called curvature noise. This occurs, when the turbulent eddies from the suction side boundary layer as well as those from the wall jet encounter a centrifugal acceleration around the convex part of the flap marked in figure 19 by the green box of the fRPM source region. Compared to the above presented results, this curvature noise radiates in a similar frequency range as the trailing edge noise from the attached flow, whereas the directivity compares better to the jet mixing noise. Here again, an effective shielding 
in upstream down direction can be observed although the sound waves are diffracted more at the airfoil's leading and trailing edge. This is most probably due to the larger wavelengths compared to the jet noise. However, also the main radiation direction is pointing downwards at an angle of about $\theta=330^{\circ}$. Hence, the shielding is less effective than for the jet mixing noise when considering community noise. In terms of sound pressure levels, the curvature noise is of a similar magnitude as the trailing edge noise. Accordingly, curvature noise levels lie in between the sound pressure levels of the attached and the partially detached flow. Other than for the jet mixing noise, this comparison is supposed to be valid as the same source modeling for the trailing edge and the curvature noise is used. A direct comparison for the two setups is presented in figure 20. It reveals that for the completely attached flow the curvature noise dominates the trailing edge noise by up to $10 \mathrm{~dB}$, depending on the radiation angle. However, in the radiation direction forward down, which is relevant for community noise, the trailing edge noise is about $5 \mathrm{~dB}$ higher. For the partially detached flow the trailing edge noise is more prominent for almost all angles. The only relevant exception concerning community noise is at $\theta=330^{\circ} \pm 10^{\circ}$. This supports again the importance of taking into account the real mean flow state when designing a low-noise high-lift system. Otherwise it might occur that applied noise reduction techniques are at least less effective.

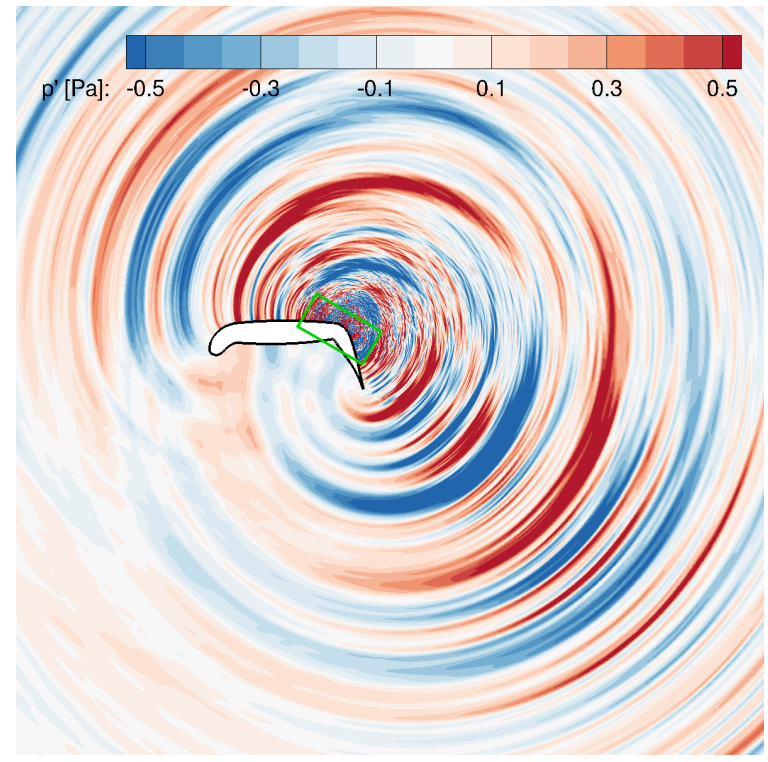

(a) Snapshot of the curvature noise sound pressure field with fRPM source region (setup 1)

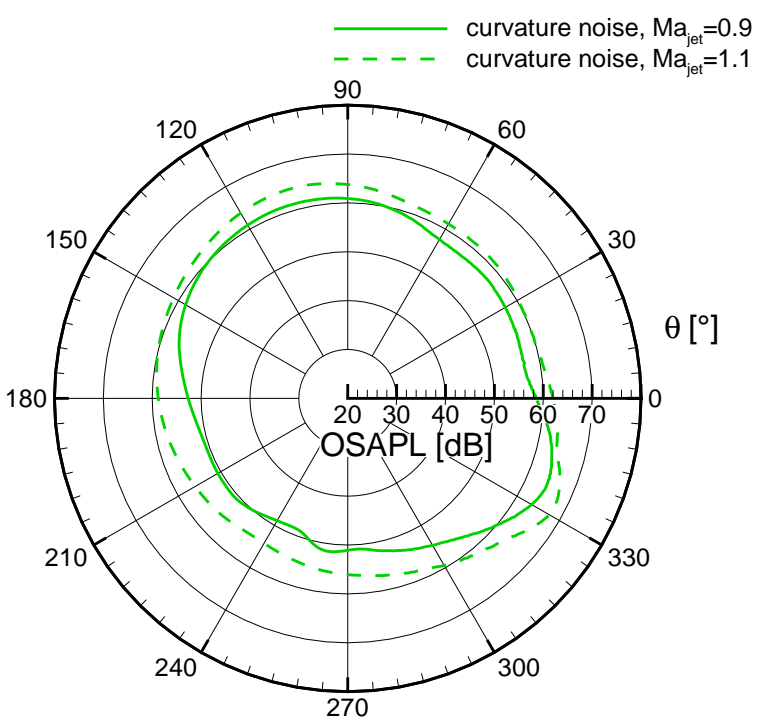

(b) Directivity of the OASPL $(300 \mathrm{~Hz}-30 \mathrm{kHz})$ at $1.5 \cdot c$ from the trailing edge for different jet velocities: $M a_{\text {jet }}=0.9$ (setup 1 , solid line), $M a_{\text {jet }}=1.1$ (setup 2, dashed line)

Figure 19: Simulation results for the curvature noise source.

For a more detailed analysis of the weighting of the trailing edge and the curvature noise sources, figure 21 depicts the recorded spectra at two different radiation angles. First thing to notice is that the increased jet velocity and angle of attack result in an increase of the sound pressure levels for the whole frequency range. For the case of completely attached flow, both spectra for the trailing edge as well as curvature noise are shown. It can be seen, that at an angle of $\theta=240^{\circ}$ the spectra are almost alike, while for $330^{\circ}$ the curvature noise dominates over the whole frequency range.

In section IV.B it was discussed that in the experiments single noise source mechanisms were separated by the application of local noise reduction treatments. As the curvature noise is not as easily accessible as the trailing edge noise, no measures were applied. However, the results showed that the trailing edge seems to be a dominant source region. From the simulations, this is only valid for the case of locally separated flow. Otherwise, the curvature noise masks noise coming from the trailing edge. This may point to different aspects, that were not fully covered by the data. On the one side, it might be that in the experiments the flow was partially separated above the attached wall jet. On the other side, it is possible that the flow simulations do not predict the same turbulence characteristics of the jet and the boundary layer that occur in the wind tunnel. Therefore, the comparison between experiments and simulations does not give clear results but very valuable insights for following investigations. Furthermore, discussing the measured jet-related noise sources, a low-frequency contribution was found that scales with $U_{j e t}^{6.5}$ which indicates a turbulence-geometry 


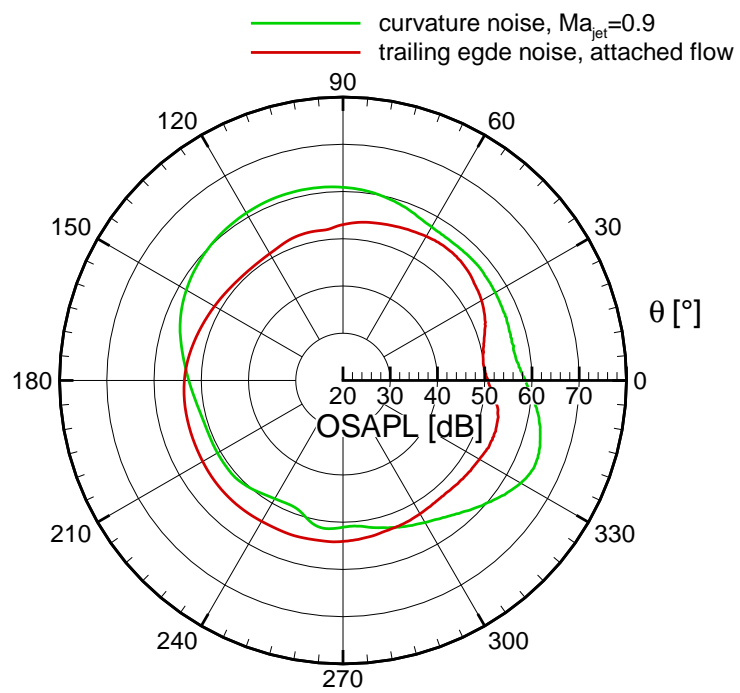

(a) $M a_{\text {jet }}=0.9, \alpha=0^{\circ}$

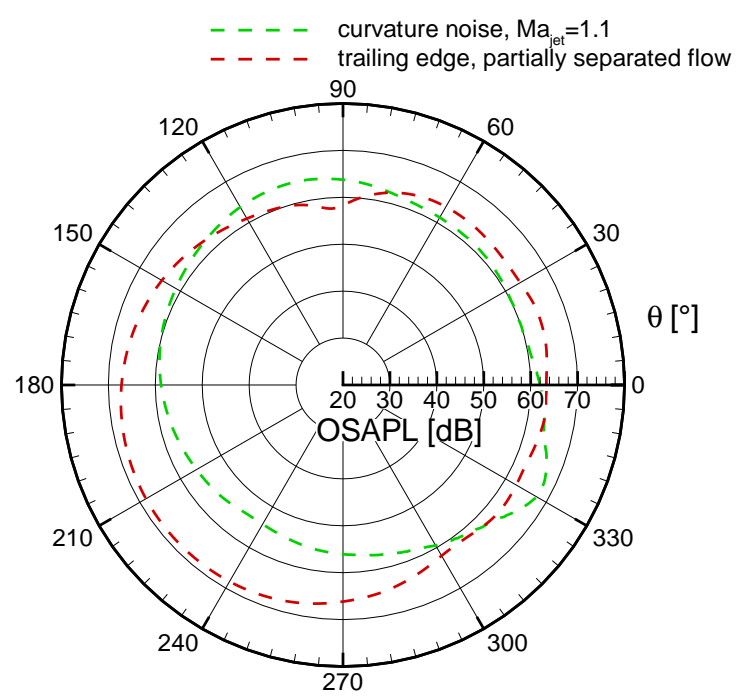

(b) $M a_{\text {jet }}=1.1, \alpha=15^{\circ}$

Figure 20: Directivity of the OASPL $(300 \mathrm{~Hz}-30 \mathrm{kHz})$ at $1.5 \cdot c$ from the trailing edge of trailing edge (red) and curvature noise (green) (setup 1: solid lines, setup 2: dashed lines).

interaction source mechanism. It is assumed, that this relates either to jet-nozzle interaction or the curvature effect. Here, further simulations will need to be run at different jet velocities to derive scaling laws.

\section{IV.D. Flap Kink Noise}

The last possible considered noise source location is found on the pressure side of the airfoil in the region of detached flow at the flap, shown by the blue box in figure 22. The radiated sound pressure is several orders of magnitude smaller in amplitude than for the other three source mechanisms (trailing edge, curvature and jet). Hence, even though this source mainly radiates in downward direction, it does not show to be a significant acoustic source. This observation is also related to the turbulence intensity and local flow velocity that is lower than at the trailing edge or the jet region. Therefore, a more detailed analysis is skipped here.

As already discussed in section IV.B a potential separation and therefore noise reducing fairing in the flap kink showed no acoustic effect. This supports the assumption that the noise emitted from the separation bubble is indeed no dominant source mechanism, as suggested by the numerical result.

\section{Summary and Outlook}

In the present paper the numerical and experimental analysis of noise radiated from an airfoil with circulation control is addressed. Valuable insights into the different acoustic source mechanisms are gained from both the numerical as well as experimental approach. The simulations are used to come up with an acoustic breakdown to single sources on the airfoil, namely jet mixing noise, trailing edge noise, curvature noise and flow separation noise. It was found that for the downward facing radiation direction, which is of special interest considering community noise, trailing edge noise and curvature noise are the dominant contributors, while the high frequency jet mixing noise radiates mostly to the top and the flow separation noise in the flap kink is much weaker. By varying the flow conditions around the airfoil it was found that partial flow separation on the flap's suction side results in a significant low-frequency noise source. Thus, although these separations may be aerodynamically acceptable to produce high lift coefficients, they should be avoided when designing a low-noise high-lift system.

On the experimental part of the work, an analysis of mainly the high speed jet related noise and the trailing edge noise is provided. Considering the jet noise, two different regimes are identified. In the lowfrequency domain presumably jet-nozzle interaction or curvature effects from the bending of the jet become dominant while for higher frequencies jet mixing noise is expected to be the driving source mechanism. 


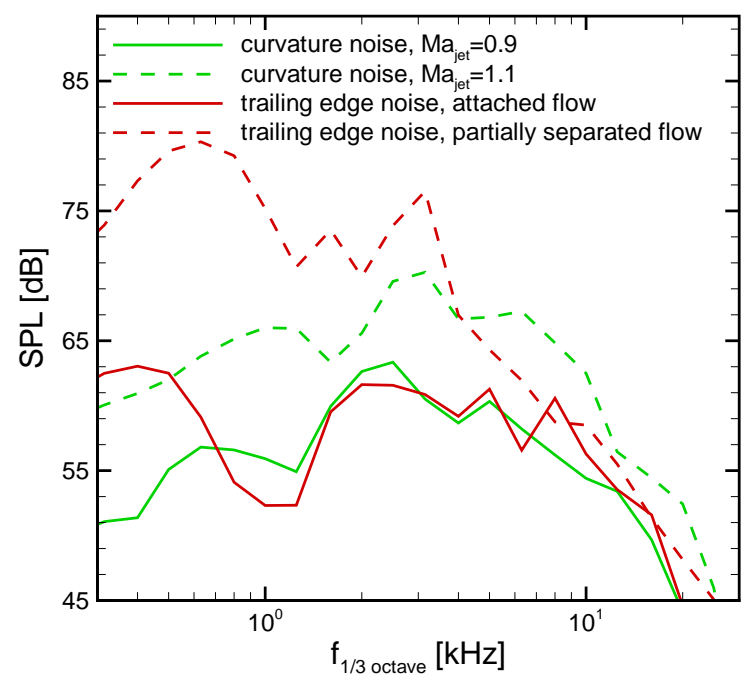

(a) Spectra recorded at an angular position of $\theta=240^{\circ}$

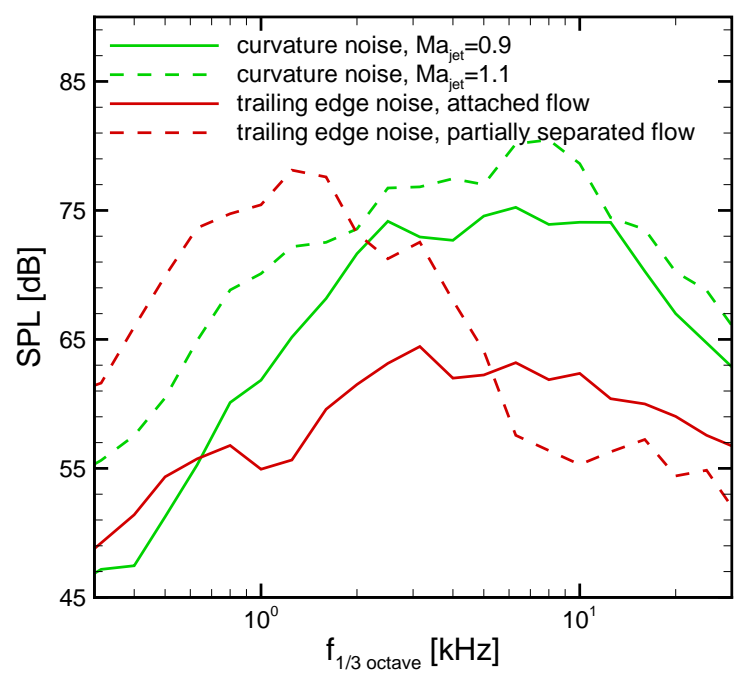

(b) Spectra recorded at an angular position of $\theta=330^{\circ}$

Figure 21: Calculated spectra for the trailing edge and curvature noise source at $1.5 \cdot c$ from the trailing edge (setup 1: solid lines, setup 2: dashed lines).

Furthermore, a first approach to noise reduction techniques is made that helped in relating the different source mechanisms. Thereby, it was shown that brushes applied to the trailing edge of the flap reduce the emitted noise for frequencies in the range of $2-10 \mathrm{kHz}$. This supports the assumption gained from the simulations, that trailing edge noise might in fact be a considerable noise source mechanism on such circulation-control airfoils.

In the future the results of this work may serve as a basis to come up with efficient noise reduction techniques, such as trailing edge devices or changes in the design of the flap. Beforehand, further analysis of different setups will be carried out both numerically and experimentally. With the presented insights into the source mechanism, it is now possible to design the experiments in order to separate the different source mechanisms. Furthermore the numerical settings can be chosen to get a close match with the experimental setup. Thereby the link between the two approaches is expected to provide data for detailed analysis. The following steps then will cope with the design of a low-noise airfoil with the application of noise reduction technology, such as trailing edge modifications.

\section{Acknowledgment}

Financial support is provided by the German Research Foundation (Deutsche Forschungsgemeinschaft, DFG) in the framework of the Collaborative Research Center 880. Computational and experimental resources are provided by the German Aerospace Center (Deutsches Zentrum für Luft- und Raumfahrt e.V., DLR), Institute of Aerodynamics and Flow Technology.

\section{References}

${ }^{1}$ Delfs, J. W., Appel, C., Bernicke, P., Blech, C., Blinstrup, J., Heykena, C., Kumar, P., Kutscher, K., Lippitz, N., Lummer, M., Rossian, L., and Savoni, L., "Aircraft and technology for low noise short take-off and landing," AIAA Paper 2017-3558, 2017.

${ }^{2}$ Coanda, H., "Procédé de propulsion dans un fluide," 1932.

${ }^{3}$ Pfingsten, K. C. and Radespiel, R., "Experimental and numerical investigations of a circulation control airfoil," AIAA Paper 2009-533, 2009.

${ }^{4}$ Loth, J. L., "Advantages of combining BLC suction with circulation control high-lift generation," Application of circulation control technology, Progress in Astronautics and Aeronautics, Vol. 214, pp. 3-21.

${ }^{5}$ Pott-Pollenske, M. and Pfingsten, K. C., "Aeroacoustic Performance of an Airfoil with Circulation Control," AIAA Paper 2010-3881, 2016.

${ }^{6}$ Howe, M. S., "Analytical Study of the Noise Generated by a Coanda Wall Jet Circulation Control Device," Final Report 


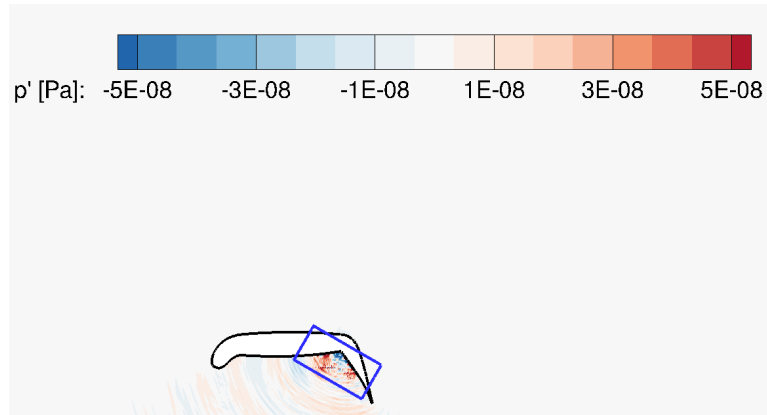

Figure 22: Snapshot of the flap kink detached flow noise sound pressure field (setup 1).

AM 00-004, 2000.

${ }^{7}$ Delfs, J. W., Bauer, M., Ewert, R., Grogger, H. A., Lummer, M., and Lauke, T. G. W., "Numerical Simulation of Aerodynamic Noise with DLR's aeroacoustic code PIANO," 2008.

${ }^{8}$ Ewert, R., Dierke, J., Siebert, J., Neifeld, A., Appel, C., Siefert, M., and Kornow, O., "CAA broadband noise prediction for aeroacoustic design," Journal of Sound and Vibration, Vol. 330, No. 17, 2011, pp. 4139-4160.

${ }^{9}$ Ewert, R., "Broadband slat noise prediction based on CAA and stochastic sound sources from a fast random particle-mesh (RPM) method," Computers \&5 Fluids, Vol. 37, No. 4, 2008, pp. 369-387.

${ }^{10}$ Tam, C. K. W. and Webb, J. C., "Dispersion-Relation-Preserving Finite Difference Schemes for Computational Acoustics," Journal of Computational Physics, Vol. 107, No. 2, 1993, pp. 262-281.

${ }^{11}$ Tam, C. K. W. and Auriault, L., "Jet Mixing Noise from Fine-Scale Turbulence," AIAA Journal, Vol. 37, No. 2, pp. 145153.

${ }^{12}$ Ewert, R., Dierke, J., and Neifeld, A., "Linear and Non-Linear Perturbation Equations with Relaxation Source Terms for Forced Eddy Simulation of Aeroacoustic Sound Generation," AIAA Paper 2014-3053, 2014.

${ }^{13}$ Appel, C., Rossignol, K.-S., Klabes, A., Neifeld, A., Herr, M., and Ewert, R., "RANS/CAA based Prediciton of Jet Mixing Noise in Cruse Flight," AIAA Paper 2014-2603, 2014.

${ }^{14}$ Ahuja, K. K., Sankar, L. N., Englar, R. J., Munro, S. E., Li, Y., and Gaeta, R. J., "Application of Circulation Control Technology to Airframe Noise Reduction," GTRI Report A5928/2003-1, 2003.

${ }^{15}$ Rautmann, C., Dierke, J., Ewert, R., Hu, N., and Delfs, J., "Generic Airfoil Trailing-Edge Noise Prediction using Stochastic Sources from Synthetic Turbulence," AIAA Paper 2014-3298, 2014.

${ }^{16}$ Herr, M., Ewert, R., Rautmann, C., Kamruzzaman, M., Bekiropoulos, D., Iob, A., Arina, R., Batten, P., Chakravarthy, S., and Bertagnolio, F., "Broadband Trailing-Edge Noise Predictions-Overview of BANC-III Results," Aiaa paper, 2015.

${ }^{17}$ Herr, M., Pott-Pollenske, M., Ewert, R., Boenke, D., Bekiropoulos, D., Siebert, J., Delfs, J., and Rudenko, A., "LargeScale Studies on Slat Noise Reduction," Aiaa paper, 2015.

${ }^{18}$ Ewert, R. and Schröder, W., "Acoustic perturbation equations based on flow decomposition via source filtering," Journal of Computational Physics, Vol. 188, No. 2, 2003, pp. 365-398.

${ }^{19}$ Neifeld, A., Boenke, D., Dierke, J., and Ewert, R., "Jet Noise Prediction with Eddy Relaxation Source Model," AIAA Paper 2015-2370, 2015.

${ }^{20}$ Neifeld, A., Appel, C., Dierke, J., Ewert, R., and Delfs, J., "CAA Prediction of Jet-Wing Interaction Noise Using an Eddy Relaxation Source Model," New Results in Numerical and Experimental Fluid Mechanics XI, 2016.

${ }^{21}$ Rossian, L., Faßmann, B. W., Ewert, R., and Delfs, J. W., "Prediction of Porous Trailing Edge Noise Reduction Using Acoustic Jump-Conditions at Porous Interfaces," AIAA Paper 2016-2920, 2016.

${ }^{22}$ Sijtsma, P., "CLEAN based on spatial source coherence," International journal of aeroacoustics, Vol. 6, No. 4, 2007, pp. $357-374$.

${ }^{23}$ Williams, J., "Some open questions on the jet noise problem," Tech. rep., BOEING SCIENTIFIC RESEARCH LABS SEATTLE WASH FLIGHT SCIENCES LAB, 1968.

${ }^{24}$ Seiner, J. M., Manning, J., and Ponton, M., "Acoustic properties associated with rectangular geometry supersonic nozzles," Thermophysical Aspects of Re-entry Flows, 1986. 